\title{
Apolarity and Direct Sum Decomposability of Polynomials
}

\author{
WERONIKA BUCZYŃSKA, JAROSŁAW BuCZYŃSKI, \\ Johannes KlePPE, \& ZaCh TEITLER
}

\begin{abstract}
A polynomial is a direct sum if it can be written as a sum of two nonzero polynomials in some distinct sets of variables, up to a linear change of variables. We analyze criteria for a homogeneous polynomial to be decomposable as a direct sum in terms of the apolar ideal of the polynomial. We prove that the apolar ideal of a polynomial of degree $d$ strictly depending on all variables has a minimal generator of degree $d$ if and only if it is a limit of direct sums.
\end{abstract}

\section{Introduction}

A homogeneous polynomial $F$ is a direct sum if there exist nonzero polynomials $F_{1}, F_{2}$ such that $F=F_{1}+F_{2}$ and $F_{1}=F_{1}\left(t_{1}, \ldots, t_{s}\right), F_{2}=F_{2}\left(t_{s+1}, \ldots, t_{n}\right)$ for some linearly independent linear forms $t_{1}, \ldots, t_{n}$. For example, $F=x y$ is a direct sum since $F=\frac{1}{4}(x+y)^{2}-\frac{1}{4}(x-y)^{2}$. In coordinate-free terms, $F \in S^{d} V$ is a direct sum if $F=F_{1}+F_{2}$ for nonzero $F_{i} \in S^{d} V_{i}, i=1,2$, such that $V_{1} \oplus V_{2}=V$.

Most polynomials are not direct sums; see Lemma 3.3. Nevertheless, it can be difficult to show that a particular polynomial is not a direct sum. For instance, S. Shafiei shared with us the following question: is the generic determinant $\operatorname{det}_{n}=$ $\operatorname{det}\left(\left(x_{i, j}\right)_{i, j=1}^{n}\right)$, a homogeneous form of degree $n$ in $n^{2}$ variables, a direct sum? For $n=2, \operatorname{det}_{2}=x_{1,1} x_{2,2}-x_{1,2} x_{2,1}$ is visibly a direct sum. On the other hand, for $n>2$, it is easy to see that the determinant is not decomposable as a direct sum in the original variables, but it is not immediately clear whether it is decomposable after a linear change of coordinates. We answer this question in the negative; see Corollary 1.2.

Problem A. Give necessary or sufficient conditions for a polynomial to be a direct sum.

Received August 10, 2013. Revision received July 14, 2015.

W. Buczyńska is supported by the research project "Rangi i rangi brzegowe wielomianów oraz równania rozmaitości siecznych" funded by Polish Financial Means for Science in 2012-2014. J. Buczyński is supported by the project "Secant varieties, computational complexity, and toric degenerations" realized within the Homing Plus programme of Foundation for Polish Science, cofinanced from European Union, Regional Development Fund. J. Buczyński is also supported by the scholarship "START" of the Foundation for Polish Science. Furthermore, while revising the article, he is supported by a scholarship of the Polish Ministry of Science. 
We approach this problem through apolarity. Suppose $S=\mathbb{C}\left[x_{1}, \ldots, x_{n}\right]$ and $T=$ $\mathbb{C}\left[\alpha_{1}, \ldots, \alpha_{n}\right]$. When the number of variables is small, we may write $S=\mathbb{C}[x, y]$ and $T=\mathbb{C}[\alpha, \beta]$, or $S=\mathbb{C}[x, y, z]$ and $T=\mathbb{C}[\alpha, \beta, \gamma]$. (For simplicity, we assume throughout that our base field is the field of complex numbers $\mathbb{C}$. However, our results also hold for other algebraically closed base fields of any characteristic. We comment on the applicable modifications in Section 6.3.) We let $T$ act on $S$ by letting $\alpha_{i}$ act as the partial differentiation operator $\partial / \partial x_{i}$. This action is denoted by the symbol $\lrcorner$, as in $\left.\alpha \beta^{2}\right\lrcorner x^{2} y^{3} z^{4}=\partial^{3} x^{2} y^{3} z^{4} / \partial x \partial y^{2}=12 x y z^{4}$. This is the apolarity action; $T$ is called the dual ring of $S$. Let $F \in S$ be a homogeneous polynomial of degree $d$. The apolar or annihilating ideal $F^{\perp} \subset T$ is the set of polynomials $\Theta \in T$ such that $\Theta\lrcorner F=0$. The quotient $A_{F}=T / F^{\perp}$ is called the apolar algebra of $F$.

The Waring rank $r(F)$ of $F$ is the least $r$ such that $F=\ell_{1}^{d}+\cdots+\ell_{r}^{d}$ for some linear forms $\ell_{i}$. A lower bound for Waring rank, following from ideas of Sylvester [53] in 1851, is that $r(F)$ is bounded below by the maximum value of the Hilbert function of $A_{F}$. Ranestad and Schreyer [43] have recently shown that the Waring rank of $F$ is bounded below by $\frac{1}{\delta}$ length $\left(A_{F}\right)$, where $\delta$ is the greatest degree of a minimal generator of $F^{\perp}$, and length $\left(A_{F}\right)$ is the length of the apolar algebra, that is, the sum of all the values of the Hilbert function of $A_{F}$. The bound of Ranestad-Schreyer is best when $\delta$ is small, that is, when $F^{\perp}$ is generated in small degrees. So it is natural to ask when this occurs or, conversely, when $F^{\perp}$ has high-degree generators.

Problem B. Give necessary or sufficient conditions for $F^{\perp}$ to be generated in low degrees or in high degrees; that is, for the greatest degree $\delta$ of a minimal generator of $F^{\perp}$ to be small or large.

As we shall see, "small" and "large" should be considered relative to the degree $d$ of $F$.

It is through serendipity that while simultaneously studying Problems A and B as separate problems, the authors noticed that they were actually not separate. These two problems are linked by the following result (see also [33, Lemma 2.9, Lemma 3.27]).

THEOREM 1.1. If $F$ is a direct sum, then $F^{\perp}$ has a minimal generator of degree $\operatorname{deg}(F)$.

Shafiei [47] has shown that the apolar ideal of the generic determinant $\operatorname{det}_{n}$ is generated in degree 2 . Thus, we have the following:

\section{CoROllary 1.2. For $n>2$, the generic determinant is not a direct sum.}

Other results of Shafiei concerning apolar ideals of permanents, Pfaffians, and so on have similar consequences for direct sum indecomposability of these forms.

Despite its centrality in linking Problems A and B, Theorem 1.1 is surprisingly easy to prove; see Section 1.2. 
The converse to Theorem 1.1 does not hold.

ExAmple 1.3. $F=x y^{2} \in S=\mathbb{C}[x, y]$ has $F^{\perp}=\left\langle\alpha^{2}, \beta^{3}\right\rangle \subset T=\mathbb{C}[\alpha, \beta]$ with the minimal generator $\beta^{3}$ of degree 3 , but $F$ is not a direct sum. Indeed, in two variables, a direct sum $x^{d}-y^{d}$ factors as $x^{d}-y^{d}=\prod_{k=1}^{d}\left(x-\zeta^{k} y\right)(\zeta$ a primitive $d$ th root of unity) with distinct linear factors, whereas $x y^{2}$ does not have distinct factors; or use Proposition 2.12.

EXAMPLE 1.4. The cubic $F=x^{2} y-y^{2} z=y\left(x^{2}-y z\right) \in \mathbb{C}[x, y, z]$ has $F^{\perp}=$ $\left\langle\gamma^{2}, \alpha \gamma, \alpha^{2}+\beta \gamma, \beta^{3}, \alpha \beta^{2}\right\rangle$, so $F^{\perp}$ has two minimal generators of degree 3 . Thus, $F$ satisfies the necessary condition of Theorem 1.1. However, $F$ is not a direct sum by Proposition 2.12 .

Note however that $x y^{2}$ is a limit of direct sums:

$$
x y^{2}=\lim _{t \rightarrow 0} \frac{1}{3 t}\left((y+t x)^{3}-y^{3}\right)
$$

as is $y\left(x^{2}+y z\right)$ :

$$
y\left(x^{2}+y z\right)=\lim _{t \rightarrow 0} \frac{1}{6 t^{2}}\left(\left(y+t x+2 t^{2} z\right)^{3}+(y-t x)^{3}-2 y^{3}\right) .
$$

We will show that if $F^{\perp}$ has a minimal generator of degree $\operatorname{deg}(F)$, then $F$ is a limit of direct sums. But the converse does not hold: not every limit $F$ of direct sums has the property that $F^{\perp}$ has a minimal generator of degree $\operatorname{deg}(F)$.

EXAMPLE 1.5. For $t \neq 0, x^{d}-t y^{d}$ is a direct sum, and $\lim _{t \rightarrow 0} x^{d}-t y^{d}=x^{d}$. However, $\left(x^{d}\right)^{\perp}=\left\langle\alpha^{d+1}, \beta\right\rangle$ has no minimal generator of degree $d$.

A perhaps more satisfying example is $\lim _{t \rightarrow 0} x y z-t w^{3}=x y z$, again a limit of direct sums, with $(x y z)^{\perp}=\left\langle\alpha^{2}, \beta^{2}, \gamma^{2}, \delta\right\rangle$, having no minimal generator of degree 3 .

It is no coincidence that in both of these examples the limit polynomial uses fewer variables than the direct sums at $t \neq 0$. We will show that in general, if $F$ is a limit of direct sums that cannot be written using fewer variables, then $F^{\perp}$ has a minimal generator of degree $\operatorname{deg}(F)$.

We now introduce terminology to give a precise statement of these results.

First, note that $F^{\perp}$ is a homogeneous ideal containing $\left\langle\alpha_{1}, \ldots, \alpha_{n}\right\rangle^{d+1}$, all forms of degree at least $d+1$. Thus, $F^{\perp}$ is generated in degree at most $d+1$ : the $\delta$ in the Ranestad-Schreyer theorem satisfies $1 \leq \delta \leq d+1$. We mention the following observation, previously noted by Casnati and Notari [13, Rem. 4.3].

Proposition 1.6. $F^{\perp}$ has a minimal generator of degree $d+1$ if and only if $F=\ell^{d}$ is a power of a linear form.

A proof is given in Section 1.2. 
For brevity, we refer to a minimal generator of $F^{\perp}$ as an apolar generator of $F$. Any apolar generator of degree equal to $\operatorname{deg}(F)$ is called an equipotent apolar generator.

We introduce the notation DirSum $=\operatorname{DirSum}_{n ; d}$ for the set of direct sums (of degree $d$ in $n$ variables), ApoEqu for the set of forms with an equipotent apolar generator, and Con for the set of forms that cannot be written using fewer variables. (Such forms are called concise; see Section 2.2.) Fix $n, d$. We will show that every form with an equipotent apolar generator is a limit of direct sums, so that we have the following inclusions:

\section{$\begin{array}{ccccc}\text { DirSum } & \subset & \text { ApoEqu } & \subset & \overline{\text { DirSum }} \\ \cup & & \cup & & \cup \\ \text { DirSum } \cap \text { Con } & \subset & \text { ApoEqu } \cap \text { Con } & \subset & \overline{\text { DirSum } \cap \text { Con }}\end{array}$}

In fact, most of these inclusions are strict in general. The vertical inclusions clearly are strict as soon as $n \geq 2$. We have DirSum $\cap$ Con $\varsubsetneqq$ ApoEqu $\cap$ Con (and of course DirSum $\varsubsetneqq$ ApoEqu) by Examples 1.3 and 1.4. We also have ApoEqu $\varsubsetneqq \overline{\text { DirSum }}$ by Example 1.5 .

Surprisingly, the last remaining inclusion is in fact an equality (compare with [33, Cor. 4.7]).

THEOREM 1.7. For $n \geq 2$ and $d \geq 3$, every form with an equipotent apolar generator is a limit of direct sums, and conversely, every concise limit of direct sums has an equipotent apolar generator. In particular, ApoEqu $\cap$ Con $=\overline{\operatorname{DirSum}} \cap \mathrm{Con}$.

This theorem is proved in Section 4.2. One direction is proved in Theorem 4.5, and the other direction is proved in Theorem 4.8. Moreover, Theorem 4.5 provides a normal form for the limits of direct sums that are not direct sums. In such cases, for some choice of basis $x_{1}, \ldots, x_{k}, y_{1}, \ldots, y_{k}, z_{1}, \ldots, z_{n-2 k}$ of $V$,

$$
\begin{aligned}
& F\left(x_{1}, \ldots, x_{k}, y_{1}, \ldots, y_{k}, z_{1}, \ldots, z_{n-2 k}\right) \\
& \quad=\sum_{i=1}^{k} x_{i} \frac{\partial H\left(y_{1}, \ldots, y_{k}\right)}{\partial y_{i}}+G\left(y_{1}, \ldots, y_{k}, z_{1}, \ldots, z_{n-2 k}\right)
\end{aligned}
$$

for homogeneous polynomials $H(y)$ in $k$ variables and $G(y, z)$ in $n-k$ variables, both of degree $d$.

One might naively hope to prove at least one direction of Theorem 1.7 by arguing that if $F_{t} \rightarrow F$, then presumably $F_{t}^{\perp} \rightarrow F^{\perp}$. If for each $t \neq 0, F_{t}$ is a direct sum, then $F_{t}^{\perp}$ has a minimal generator of degree $d=\operatorname{deg} F$ by Theorem 1.1; then one might hope to finish by appealing to the semicontinuity of graded Betti numbers (see Section 2.6) to show that $F^{\perp}$ also has at least one minimal generator of degree $d$. However, this argument cannot succeed since $F_{t} \rightarrow F$ does not imply $F_{t}^{\perp} \rightarrow F^{\perp}$ as a flat limit. For instance, consider the family of polynomials $F_{t}=t x^{d}+x y^{d-1}$ in $x$ and $y$ parameterized by $t$, with $d \geq 4$. We have 
$F_{t} \rightarrow F_{0}=x y^{d-1}$ and

$$
F_{t}^{\perp}= \begin{cases}\left\langle\alpha^{2} \beta, \alpha^{d-1}+d t \beta^{d-1}\right\rangle & \text { for } t \neq 0, \text { or } \\ \left\langle\alpha^{2}, \beta^{d}\right\rangle & \text { for } t=0 .\end{cases}
$$

Thus, the flat $\operatorname{limit}_{\lim _{t \rightarrow 0}}\left(F_{t}^{\perp}\right)=\left\langle\alpha^{2} \beta, \alpha^{d-1}, \beta^{d}\right\rangle \varsubsetneqq F_{0}^{\perp}$.

Nevertheless, for those cases in which $F_{t} \rightarrow F$, the $F_{t}$ are direct sums, and $F_{t}^{\perp} \rightarrow F^{\perp}$ is a flat family, it follows that $F^{\perp}$ has a degree $d$ generator by semicontinuity. When such a family $\left\{F_{t}\right\}$ exists, we say $F$ is an apolar limit of direct sums. The locus of apolar limits of direct sums is denoted ApoLim. We have the following:

TheOrem 1.8. ApoLim $\subset$ ApoEqu, and:

(i) For $n=14$ and $d \geq 28$, the inclusion is strict: ApoLim $\varsubsetneqq$ ApoEqu.

(ii) If $d=3$ or if $n=3$, then ApoLim $=$ ApoEqu.

The inclusion ApoLim $\subset$ ApoEqu follows by the semicontinuity of graded Betti numbers, as described before. The strictness of the inclusion for $n=14$ is explained in Section 5.2, particularly, in Proposition 5.17. The proof of the equality for $d=3$ is straightforward, and it is explained in Proposition 5.2. The proof of the equality for $n=3$ is obtained by longer but elementary methods in Theorem 5.19. Certainly, using more refined techniques, we may be able to determine whether ApoLim = ApoEqu also in other cases.

The strictness of the inclusion is a consequence of the existence of certain zero-dimensional Gorenstein local schemes with restricted deformations, which we call uncleavable schemes. A scheme supported at a single point is uncleavable if all its deformations are supported at a single point; see Section 5.2 for references and more details. We show that for at least $n=14$, we have ApoLim $\varsubsetneqq$ ApoEqu. This is because the shortest nonsmoothable zero-dimensional Gorenstein scheme of length 14 is uncleavable. However, we expect (but are not able to prove) that ApoLim $\varsubsetneqq$ ApoEqu should hold for all sufficiently large $n$. We explain in detail in Section 5.2 which deformation-theoretic properties of schemes of length $n$ we need in order to obtain ApoLim $\neq$ ApoEqu.

In any case, we emphasize that because of Theorem 1.8, the naive hope described before cannot suffice to prove Theorem 1.7. That is, for some $n$ and $d$, there are forms in ApoEqu whose apolar generators of degree $d$ do not arise via semicontinuity of graded Betti numbers for any family of direct sums. The strictness of ApoLim $\varsubsetneqq$ ApoEqu forces a more delicate argument for Theorem 1.7.

See also [33, Sect. 4.2, Cor. 4.24] for related examples.

It is also interesting to study the case in which $F^{\perp}$ is generated in low degrees. For example, $\left(\operatorname{det}_{n}\right)^{\perp}$ is generated in degree 2 , as is $\left(x_{1} \cdots x_{n}\right)^{\perp}=\left\langle\alpha_{1}^{2}, \ldots, \alpha_{n}^{2}\right\rangle$. See Table 1 for examples of plane cubics. We show that an upper bound for the degrees of minimal generators of $F^{\perp}$ forces an upper bound on the degree of $F$; equivalently, if $F$ has a high degree relative to the number of variables, then $F^{\perp}$ must have at least one high-degree minimal generator. 
THEOREM 1.9. If $F$ is a homogeneous form of degree $d$ in $n$ variables and $\delta$ is the highest among the degrees of minimal generators of $F^{\perp}$, then $d \leq(\delta-1) n$.

In particular, if $F^{\perp}$ is generated by quadrics, then $d \leq n$. It would be interesting to classify polynomials $F$ of degree $d=n$ such that $F^{\perp}$ is generated by quadrics. See Section 4.3 for a brief discussion and the proof of the theorem.

Notation 1.10. Throughout the paper, $F \in S^{d} V$ is a homogeneous form of degree $d$ in $n=\operatorname{dim} V$ variables. More generally, $F$ may be a divided-powers form of degree $d$; see [29, App. A].

REMARK 1.11. Direct sums and their limits have also appeared in other articles. In [54], functions (not necessarily polynomials) are called decomposable when they are sums of functions in independent variables. In [40], they are called sum-maps, whereas in [39] and [16], they are called direct sums. In [55], polynomials with a direct sum decomposition are called polynomials of Sebastiani-Thom type. They are called connected sums in [47], following [37; 51], where the term connected sum is used to refer to a closely related concept; see Section 2.8. In [44], forms (homogeneous polynomials) $p$ and $q$ over $\mathbb{C}$ are called unitarily disjoint if they depend on disjoint sets of variables, after a unitary linear change of variables with respect to a fixed Hermitian product on the space of linear forms (see [44] for details). In [33], direct sum decompositions are called regular splittings, and limits of direct sum decompositions are called degenerate splittings.

In [20] and references therein, the authors study apolar algebras of homogeneous forms $F$ that are either direct sums $F=x^{d}+G\left(y_{1}, \ldots, y_{n-1}\right)$ of "type" $(1, n-1)$ or their limits $x y^{d-1}+G\left(y, z_{1}, \ldots, z_{n-2}\right)$-compare with the normal form (1) for $k=1$. Their work is motivated by earlier articles [30;21], where the special case of $n=4$ has been studied. In this series of articles, the direct sums and their limits serve the purpose of a classification of Gorenstein Artin algebras with prescribed invariants. We believe that our results in this article may have similar applications.

\subsection{Outline of Paper}

In the remainder of this Introduction, we give proofs of some elementary statements including Theorem 1.1 and Proposition 1.6.

In Section 2, we review background, including apolarity, conciseness, secant varieties, and border rank, the easy cases of binary forms and plane cubics, semicontinuity of graded Betti numbers, Gorenstein Artin algebras, and connected sums.

In Section 3, we discuss the dimension of the direct sum locus and uniqueness of direct sum decompositions.

In Section 4, we collect results that relate quadratic apolar generators to direct sums and to equipotent apolar generators. We prove Theorem 1.7. Then we prove Theorem 1.9.

In Section 5, we prove Theorem 1.8. 
In Section 6, we generalize some of our results to vector spaces of forms (linear series). We consider "overlapping sums". Finally, we discuss the generalization of our results to algebraically closed fields in any characteristic.

\subsection{Equipotent Apolar Fenerator of a Direct Sum}

We begin with a few elementary statements.

Proof of Theorem 1.1. Let $F=G-H$ where $G \in S^{x}=\mathbb{C}\left[x_{1}, \ldots, x_{i}\right], H \in S^{y}=$ $\mathbb{C}\left[y_{1}, \ldots, y_{j}\right]$, and $G, H \neq 0$. Let us denote the dual rings $T^{\alpha}=\mathbb{C}\left[\alpha_{1}, \ldots, \alpha_{i}\right]$, $T^{\beta}=\mathbb{C}\left[\beta_{1}, \ldots, \beta_{j}\right]$. We work in $S=S^{x} \otimes S^{y}=\mathbb{C}\left[x_{1}, \ldots, x_{i}, y_{1}, \ldots, y_{j}\right]$ with dual ring $T=T^{\alpha} \otimes T^{\beta}=\mathbb{C}\left[\alpha_{1}, \ldots, \alpha_{i}, \beta_{1}, \ldots, \beta_{j}\right]$.

We have $G^{\perp} \cap H^{\perp} \subset F^{\perp}$, where $G^{\perp}$ and $H^{\perp}$ are computed in $T$ rather than $T^{\alpha}, T^{\beta}$. On the other hand, if $\Theta \in\left(F^{\perp}\right)_{k}$, then $\left.\left.\Theta\right\lrcorner G=\Theta\right\lrcorner H \in S_{d-k}^{x} \cap S_{d-k}^{y}$. But this intersection is zero if $k \neq d$, so we must have $\Theta\lrcorner G=\Theta\lrcorner H=0$. Thus, $\left(G^{\perp} \cap H^{\perp}\right)_{k}=\left(F^{\perp}\right)_{k}$ for all $k \neq d$.

Now let $\delta_{1} \in T_{d}^{\alpha}$ be such that $\left.\delta_{1}\right\lrcorner G=1$, and let $\delta_{2} \in T_{d}^{\beta}$ be such that $\left.\delta_{2}\right\lrcorner H=1$. Such elements exist in abundance: there is an affine hyperplane of them in $T_{d}^{\alpha}$ and in $T_{d}^{\beta}$ by the hypothesis that $G$ and $H$ are nonzero. Let $\Delta=\delta_{1}+\delta_{2}$. Then $\left.\left.\Delta\right\lrcorner G=\Delta\right\lrcorner H=1$, so $\Delta \notin G^{\perp} \cap H^{\perp}$, but $\left.\Delta\right\lrcorner F=0$.

This element $\Delta$ is a minimal generator of $F^{\perp}$ : it cannot be generated in lower degrees, since all elements in lower degrees lie in $G^{\perp} \cap H^{\perp}$.

For future reference, we record the additional details given in the previous proof (see also [33, Lemma 3.27]).

Lemma 1.12. Let $F=G-H$ be a direct sum decomposition of degree $d$ with nonzero $G$ and $H$. Then

$$
F^{\perp}=G^{\perp} \cap H^{\perp}+\langle\Delta\rangle,
$$

where $\Delta=\delta_{1}+\delta_{2} \in T_{d}$ is homogeneous of degree $\left.\left.d, \delta_{1}\right\lrcorner G=\delta_{2}\right\lrcorner H=1, \delta_{1}$ can be written only using variables dual to variables of $G$, and $\delta_{2}$ can be written only using variables dual to variables of $H$.

Proof. The only statement left to prove is that any degree $d$ element of $F^{\perp}$ is in the ideal $G^{\perp} \cap H^{\perp}+\langle\Delta\rangle$. Let $\Theta \in\left(F^{\perp}\right)_{d}$. Then $\left.\left.\Theta\right\lrcorner G=\Theta\right\lrcorner H \in \mathbb{C}$; call this value $c$. We have $\Theta-c \Delta \in G^{\perp} \cap H^{\perp}$.

See also [12, Lemma 3.1] for a description of $F^{\perp}$ in terms of the extensions of the ideals $G^{\perp} \cap T^{\alpha}, H^{\perp} \cap T^{\beta}$.

We call $F$ an $s$-fold direct sum when $F$ can be written as a direct sum of $s$ terms, that is, $F=F_{1}+\cdots+F_{s}$ with each $F_{i} \in V_{i}$ and $V_{1} \oplus \cdots \oplus V_{s}=V$.

COROLlary 1.13. If $F=F_{1}+\cdots+F_{s}$ is an $s$-fold direct sum, then $F^{\perp}$ has at least $s-1$ equipotent apolar generators. 
Proof. It follows by induction on $s$ from Lemma 1.12. Explicitly, if $F=F_{1}+$ $\cdots+F_{s}$, then

$$
\begin{aligned}
\operatorname{dim} F_{d}^{\perp} & =1+\operatorname{dim}\left(F_{1}^{\perp} \cap\left(F_{2}+\cdots+F_{s}\right)^{\perp}\right)_{d} \\
& =\cdots=s-1+\operatorname{dim}\left(F_{1}^{\perp} \cap \cdots \cap F_{s}^{\perp}\right)_{d},
\end{aligned}
$$

so $F^{\perp}$ has at least $s-1$ minimal generators of degree $d$.

We will use frequently the following simple characterization of direct sums.

Corollary 1.14. Let $V=V_{1} \oplus V_{2}$ and $F \in S^{d} V$. Then the following are equivalent:

(i) $F=F_{1}+F_{2}$ where $F_{1} \in S^{d} V_{1}$ and $F_{2} \in S^{d} V_{2}$ (possibly, $F_{1}=0$ or $\left.F_{2}=0\right)$,

(ii) $V_{1}^{*} V_{2}^{*} \subset F^{\perp}$,

(iii) $V_{1} \cup V_{2}$ contains the common affine scheme-theoretic zero locus $V\left(\left(F^{\perp}\right)_{2}\right)$ of the quadrics in $F^{\perp}$.

If furthermore $V_{1}, V_{2} \neq 0$ and $F^{\perp}$ has no linear generators, then conditions (i)(iii) imply that $F_{1}, F_{2} \neq 0$ and $F=F_{1}+F_{2}$ is a direct sum decomposition.

Proof. If $F=F_{1}+F_{2}$, then clearly the reducible quadrics in $V_{1}^{*} V_{2}^{*}$ annihilate $F$. In the other direction, if $V_{1}^{*} V_{2}^{*} \subset F^{\perp}$, and we give $V_{1}$ a basis $x_{1}, \ldots, x_{a}$ and $V_{2}$ a basis $y_{1}, \ldots, y_{n-a}$, then condition (ii) implies that $F$ cannot have mixed terms divisible by $x_{i} y_{j}$. Thus, $F$ is as in (i).

Observe that (iii) is simply a geometric rephrasing of (ii), using the correspondence between ideals and affine schemes.

Finally, if, say, $F_{1}=0$ and $V_{1} \neq 0$, then $V_{1}^{*} \subset F^{\perp}$ gives linear generators in $F^{\perp}$.

We give an alternate proof of the statement observed by Casnati and Notari [13, Rem. 4.3] that a form $F$ of degree $d$ has an apolar generator of degree $d+1$ if and only if $F=x^{d}$ has Waring rank 1. This follows also from [29, Prop. C.33]. Our proof illustrates in a simple case some of the techniques we will use later.

Proof of Proposition 1.6. If $F=x_{1}^{d}$, then $F^{\perp}=\left\langle\alpha_{1}^{d+1}, \alpha_{2}, \ldots, \alpha_{n}\right\rangle$.

Conversely suppose $F$ has degree $d$ and $F_{d+1}^{\perp}$ has a minimal generator. Let $I=\left(F^{\perp}\right)_{\leq d}$, the ideal generated by forms in $F^{\perp}$ of degree at most $d$, and note that $I_{d+1} \subset T_{d+1}=F_{d+1}^{\perp}$ has codimension at least 1 because otherwise no generator would be needed. Then there is a nonzero polynomial $G$ of degree $d+1$ annihilated by $I$ since $G$ is annihilated by $I$ if and only if it is annihilated by $I_{d+1}$ (see [8, Prop. 3.4(iii)]). Moreover, $I_{d}=\left(F^{\perp}\right)_{d}$ has codimension exactly 1 since $I_{d}=\langle F\rangle^{\perp}$ is the annihilator of the span of $F$, which has dimension 1 . Now $I_{d} \subset\left(G^{\perp}\right)_{d} \varsubsetneqq T_{d}$, so $I_{d}=\left(G^{\perp}\right)_{d}$. Thus, the Hilbert function of $A_{G}$ has $h_{A_{G}}(d)=1$. By the symmetry of the Hilbert function of $A_{G}$ (see Section 2.7) we have $h_{A_{G}}(1)=1$. Then $G$ has only one essential variable (see Section 2.2), so $G$ can be written as a homogeneous form of a single variable; necessarily, $G$ has Waring rank 1. Say $G=x^{d+1}$. We have $G^{\perp} \subset F^{\perp}$ since $\left(G^{\perp}\right)_{\leq d}=I_{\leq d}=\left(F^{\perp}\right)_{\leq d}$ and $\left(F^{\perp}\right)_{\geq d+1}=T_{\geq d+1}$. So $\left.F=\alpha\right\lrcorner G=c x^{d}$ for some 
$\alpha$ by Lemma 2.1, that is, by the inclusion-reversing part of the Macaulay inverse system theorem.

An alternative proof, following a suggestion from the referee, applies Gotzmann's persistence theorem, [25, Thm. 3.8] or [8, Cor. 5.3], to the consecutive values $h_{T / I}(d)=h_{T / I}(d+1)=1$ to deduce that $I$ is the ideal of a point $\{x\}$ and hence $F=x^{d}$ by the apolarity lemma; see, for example, [29, Thm. 5.3]. This is similar to the proof of [29, Prop. C.33].

See also a generalization in Proposition 6.4.

\section{Background}

For a homogeneous ideal $I$ in the polynomial ring $S$, a minimal generator of $I$ is a nonzero homogeneous element of the graded module $I / \mathfrak{m} I$ where $\mathfrak{m}=$ $\left\langle x_{1}, \ldots, x_{n}\right\rangle$ is the irrelevant ideal. By the "number" of minimal generators of a given degree $k$ we mean the dimension of the $k$ th graded piece $(I / \mathfrak{m} I)_{k}$.

Following the convention of [26], by an (algebraic) variety we always mean an irreducible algebraic set. By a general element of an algebraic variety we always mean any element of some suitably chosen open dense subset.

When $V$ is a vector space, $\mathbb{P} V$ denotes the projective space of lines through the origin of $V$. When $v \in V$ is a nonzero vector, $[v]$ denotes the point in $\mathbb{P} V$ determined by $v$, that is, the line through the origin of $V$ spanned by $v$.

\subsection{Apolarity}

Let $S$ be a polynomial ring, and $T$ its dual ring. For a fixed homogeneous $F \in S_{d}$ of degree $d$, the $i$ th catalecticant $C_{F}^{i}$ is a linear map $T_{d-i} \rightarrow S_{i}$ defined by $\left.C_{F}^{i}(\Theta)=\Theta\right\lrcorner F$. The term "catalecticant" was introduced by Sylvester [53] in 1851. The images of the catalecticants are the inverse systems studied by Macaulay [36].

The catalecticant maps give an isomorphism between $A_{G}=T / G^{\perp}$ and the principal $T$-submodule of $S$ generated by $G$, consisting of elements $\Theta\lrcorner G$ for $\Theta \in T$.

Lemma 2.1. Suppose $F, G \in S$ are two homogeneous polynomials. If $G^{\perp} \subset F^{\perp}$, then $F=\Theta\lrcorner G$ for some $\Theta \in T$.

Indeed, by the inclusion-reversing part of [18, Thm. 21.6] the $T$-submodule of $S$ generated by $F$ is contained in the $T$-submodule generated by $G$.

One connection between apolarity and geometry is indicated by Exercise 21.6 of [18], which relates the apolar ideals of plane conics to their ranks. Another connection is given by the following well-known lemma (see, e.g., [17, Prop. 4.1]).

Lemma 2.2. Let $\alpha \in T_{1}$ be a linear form. Then $\alpha^{k} \in F^{\perp}$ if and only if $F$ vanishes to order at least $d-k+1$ at the corresponding point in the projective space $[\alpha] \in \mathbb{P} T_{1} \cong \mathbb{P} S_{1}^{*}$. 
In particular, $\alpha^{d-1} \in F^{\perp}$ if and only if $V(F)$ is singular at $[\alpha]$.

Proof of Lemma 2.2. $\alpha^{k} \in F^{\perp}$ is equivalent to $\left.\Theta \alpha^{k}\right\lrcorner F=0$ for all $\Theta \in T_{d-k}$, equivalently, $\left.\left.\alpha^{k}\right\lrcorner(\Theta\lrcorner F\right)=0$ for all $\Theta \in T_{d-k}$. For such $\Theta, \Theta F$ is a form of degree $k$, so $\left.\left.\alpha^{k}\right\lrcorner(\Theta\lrcorner F\right)$ is equal to the evaluation of $\left.\Theta\right\lrcorner F$ at the point $[\alpha]$ (up to scalar multiple). This vanishes for all $\Theta \in T_{d-k}$ precisely when $F$ vanishes at $[\alpha]$ to order at least $d-k+1$.

More detailed treatments of apolarity may be found in [23, Lect. 8], [29, Sect. 1.1], and [8].

\subsection{Conciseness}

A homogeneous form $F \in \mathbb{C}\left[x_{1}, \ldots, x_{n}\right]$ is concise (with respect to $x_{1}, \ldots, x_{n}$ ) if $F$ cannot be written as a polynomial in fewer variables. That is, if there are linearly independent linear forms $t_{1}, \ldots, t_{k}$ such that $F \in \mathbb{C}\left[t_{1}, \ldots, t_{k}\right] \subset$ $\mathbb{C}\left[x_{1}, \ldots, x_{n}\right]$, then $k=n$. In coordinate-free terms, $F \in S^{d} V$ is concise (with respect to $V$ ) if $F \in S^{d} W$ with $W \subset V$ implies $W=V$.

Concise polynomials are also called nondegenerate, but we will follow the terminology of the tensor literature.

The following are equivalent:

(i) $F \in S^{d} V$ is concise.

(ii) The hypersurface $V(F) \subset \mathbb{P} V^{*}$ is not a cone.

(iii) There is no point in $\mathbb{P} V^{*}$ at which $F$ vanishes to order $d$.

(iv) The catalecticant $C_{F}^{1}$ is onto.

(v) The apolar ideal $F^{\perp}$ has no linear elements: $F_{1}^{\perp}=0$.

We define the span of $F$, denoted $\langle F\rangle$, to be the image of the catalecticant $C_{F}^{1}$. We have $F \in S^{d}\langle F\rangle$. With this notation, $G+H$ is a direct sum decomposition if and only if $\langle G\rangle \cap\langle H\rangle=\{0\}$ and $G, H \neq 0$. The elements of $\langle F\rangle$ are called essential variables of $F$; by the number of essential variables of $F$ we mean the dimension $\operatorname{dim}\langle F\rangle$ of $\langle F\rangle$ as a $\mathbb{C}$-vector space. See [10].

The locus in $S^{d} V$ of nonconcise polynomials is a Zariski-closed subset called the subspace variety and denoted Sub. Its complement is the open set Con.

\subsection{Secant Varieties and Border Rank}

Let $v_{d}: \mathbb{P} V \rightarrow \mathbb{P}\left(S^{d} V\right)$ be the Veronese map, $v_{d}([\ell])=\left[\ell^{d}\right]$.

Recall that $F \in S^{d} V$ has Waring rank $r$ if and only if $F$ is a sum of $r d$ th powers of linear forms in $V$, but not fewer. Equivalently, $[F] \in \mathbb{P}\left(S^{d} V\right)$ lies in the linear span of some $r$ points in the Veronese variety $v_{d}(\mathbb{P} V)$ but does not lie in the span of any fewer points. The Zariski closure of the set of projective points corresponding to affine points of rank at most $r$ is the $r$ th secant variety $\sigma_{r}\left(v_{d}(\mathbb{P} V)\right)$ of the Veronese variety. The border rank of $F$, denoted $\operatorname{br}(F)$, is the least $k$ such that $[F]$ lies in the $k$ th secant variety of the Veronese variety. Evidently, $\operatorname{br}(F) \leq r(F)$, and strict inequality may occur. 
Note that $\operatorname{dim}\langle F\rangle \leq \operatorname{br}(F)$. Indeed, $\operatorname{dim}\langle F\rangle \leq r(F)$ clearly, so $\operatorname{dim}\langle F\rangle \leq r$ for all $F$ in a dense subset of $\sigma_{r}\left(v_{d}(\mathbb{P} V)\right)$. Since $\operatorname{dim}\langle F\rangle=\operatorname{rank} C_{F}^{1}$ varies lower semicontinuously in $F$, we have $\operatorname{dim}\langle F\rangle \leq r$ for all $F$ in $\sigma_{r}\left(v_{d}(\mathbb{P} V)\right)$.

The second secant variety $\sigma_{2}\left(v_{d}(\mathbb{P} V)\right)$ is the disjoint union of the set of points of rank 2, the set $v_{d}(\mathbb{P} V)$ itself, and (for $d>2$ ) the set of points on tangent lines to $v_{d}(\mathbb{P} V)$. Points of the third type have border rank 2, so only two essential variables. Such a point necessarily has the form $x y^{d-1}$ after a linear change of variables; we have $r\left(x y^{d-1}\right)=d$. Thus, $\operatorname{br}(F)=2$ if and only if either $F=$ $x^{d}+y^{d}$ and $r(F)=2$, or $F=x y^{d-1}$ and $r(F)=d$.

We remark that the extreme case of direct sum, that is, the $n$-fold direct sum in an $n$-dimensional vector space $V$ coincides with a sufficiently general element of the $n$th secant variety $\sigma_{n}\left(v_{d}(\mathbb{P} V)\right)$. In particular, the closure of the set of such extreme direct sums is equal to this secant variety.

\subsection{Binary Forms}

The following lemma is standard; see, for example, [29, Thm. 1.44].

Lemma 2.3. The apolar ideal of a homogeneous binary form $F$ of degree $d$ is a complete intersection ideal generated in degrees $r$ and $d+2-r$ for some integer $1 \leq r \leq(d+2) / 2$. The border rank of $F$ is $r$.

COROLlaRY 2.4. Let $F$ be a binary form of degree $d$. The apolar ideal of $F$ has a generator of degree $d$ if and only if $F$ has border rank 2 .

Note that the condition $\operatorname{br}(F)=2$ excludes polynomials of rank 1 , so $F$ must be concise. Thus the locus of concise forms with an equipotent degree apolar generator is exactly the locus of concise forms that are limits of direct sums, that is, ApoEqu $\cap$ Con $=\overline{\text { DirSum }} \cap$ Con. This is the case $n=2$ of Theorem 1.7.

\subsection{Plane Cubics}

If a plane cubic $F$ is a direct sum, then in suitable coordinates, we may write $F=x^{3}+G(y, z)$, where $G$ is a nonzero binary cubic form. We may choose coordinates so that $G(y, z)$ is $y^{3}, y^{3}+z^{3}$, or $y^{2} z$, that is, $r(G)=1$, 2, or 3 . Thus, up to change of coordinates, there are exactly three plane cubics that are direct sums.

We summarize the types of plane cubics in Table 1, adapted from [35]. The columns mean the following: $\beta_{1, i}$ is the number of apolar generators of degree $i$, $r$ is the Waring rank, and $b r$ is the border rank. (We omit $\beta_{1,4}=1$ for $F=x^{3}$.) The rows representing direct sums are in boldface, and the rows representing nonconcise polynomials are in italic face.

This table shows the case $n=3, d=3$ of Theorem 1.7.

COROLlaRY 2.5. Let $F$ be a concise plane cubic. The apolar ideal of $F$ has a minimal generator of degree 3 if and only if $F$ is a limit of direct sums. 
Table 1 Plane cubic curves

\begin{tabular}{lcccccc}
\hline \hline Description & normal form & $\beta_{1,1}$ & $\beta_{1,2}$ & $\beta_{1,3}$ & $r$ & $b r$ \\
\hline triple line & $x^{3}$ & 2 & 0 & 0 & 1 & 1 \\
three concurrent lines & $x^{3}-y^{3}$ & 1 & 1 & 1 & 2 & 2 \\
double line + line & $x^{2} y$ & 1 & 1 & 1 & 3 & 2 \\
irreducible (Fermat) & $x^{3}+y^{3}+z^{3}$ & & 3 & 2 & 3 & 3 \\
irreducible & $y^{2} z-x^{3}-x z^{2}$ & & 3 & 0 & 4 & 4 \\
cusp & $y^{2} z-x^{3}$ & 3 & 2 & 4 & 3 \\
triangle & $x y z$ & 3 & 0 & 4 & 4 \\
conic + transversal line & $x\left(x^{2}+y z\right)$ & 3 & 0 & 4 & 4 \\
irreducible, smooth & $y^{2} z-x^{3}-a x z^{2}-z^{3}$ & & 3 & 0 & 4 & 4 \\
$\quad\left(a^{3} \neq 0,-27 / 4\right)$ & & & & & \\
irreducible, singular & $y^{2} z-x^{3}-a x z^{2}-z^{3}$ & & 3 & 0 & 4 & 4 \\
$\quad\left(a^{3}=-27 / 4\right)$ & $y\left(x^{2}+y z\right)$ & 3 & 2 & 5 & 3 \\
conic + tangent line & & & & & & \\
\hline \hline
\end{tabular}

Proof. Table 1 shows that a concise plane cubic has a minimal apolar generator of degree 3 if and only if the cubic has border rank 3 , which is equivalent to its being a limit of Fermat cubics.

\subsection{Semicontinuity of Graded Betti Numbers}

In this section, we work over an arbitrary algebraically closed field $\mathbb{k}$. Let $I$ be a homogeneous ideal in a polynomial ring $T=\mathbb{k}\left[\alpha_{1}, \ldots, \alpha_{n}\right]$ with standard grading. The graded Betti numbers of $I$ are defined as follows. Fix a minimal free resolution of $T / I$,

$$
0 \leftarrow T \leftarrow \bigoplus T(-j)^{\beta_{1, j}} \leftarrow \bigoplus T(-j)^{\beta_{2, j}} \leftarrow \cdots .
$$

The $\beta_{i, j}$ are the graded Betti numbers of $I$ (more precisely, of $T / I$ ). We have $\beta_{i, j}=\operatorname{dim}_{\mathbb{k}} \operatorname{Tor}^{i}(I, \mathbb{k})_{j}[19$, Prop. 1.7].

In the proof of Theorem 4.8, we will use the fact that when the ideal $I$ varies in a flat family, the graded Betti numbers vary upper-semicontinuously. That is, if $I_{t}$ is a flat family of ideals, then $\beta_{i, j}\left(I_{0}\right) \geq \lim _{t \rightarrow 0} \beta_{i, j}\left(I_{t}\right)$.

Boratyński and Greco [5] proved that when the ideal $I$ varies in a flat family, the Hilbert functions and Betti numbers vary semicontinuously. Ragusa and Zappalá [42, Lem. 1.2] proved the semicontinuity of graded Betti numbers of flat families of zero-dimensional ideals. Semicontinuity of graded Betti numbers more generally seems to be a well-known "folk theorem"; for example, different ideas for proofs are sketched in [41, Remark following Thm. 1.1] and in [34, Cor. 3.3]. We give a quick proof here for the sake of self-containedness. 
Proposition 2.6. Let $T=\mathbb{k}\left[\alpha_{1}, \ldots, \alpha_{n}\right]$ with standard grading, and consider the power series ring $\mathbb{k}[[U]]$ with $\operatorname{deg} U=0$. Suppose that $I \subset T \otimes_{\mathbb{k}} \mathbb{k}[[U]]$ is a homogeneous ideal, flat over $\operatorname{Spec}(\mathbb{k}[[U]])$. For $\mathfrak{p} \in \operatorname{Spec}(\mathbb{k}[[U]])$, let $I_{\mathfrak{p}}=I \otimes$ $k(\mathfrak{p})$. Fix any $i$ and $j$. Then the function $\mathfrak{p} \mapsto \beta_{i, j}\left(I_{\mathfrak{p}}\right)$ is upper-semicontinuous.

Proof. Start with the Koszul resolution of $\mathbb{k}=T /\left(\alpha_{1}, \ldots, \alpha_{n}\right)$, regarded as a sheaf on $\operatorname{Spec}(\mathbb{k}[[U]])$ (although independent of $U$ ). Tensor the resolution with $I$, take the degree $j$ part of the resulting complex, and denote by $I_{k, \mathfrak{p}}$ the $k$ th graded piece of $I_{\mathfrak{p}}$. The Tor we are interested in is the homology of this complex of vector spaces:

$\cdots \leftarrow \bigwedge^{i-1} V^{*} \otimes I_{j-i+1, \mathfrak{p}} \leftarrow \bigwedge^{i} V^{*} \otimes I_{j-i, \mathfrak{p}} \leftarrow \bigwedge^{i+1} V^{*} \otimes I_{j-i-1, \mathfrak{p}} \leftarrow \cdots$,

where $V^{*}$ is the vector space spanned by $\alpha_{1}, \ldots, \alpha_{n}$. By [18, Exer. 20.14], the dimensions of the vector spaces $I_{q, \mathfrak{p}}$ are locally (in $\mathfrak{p}$ ) constant. Locally in $\mathfrak{p}$, then, this is a complex of fixed finite-dimensional vector spaces with differentials given by matrices whose entries are polynomial in $\mathfrak{p}$. The graded Betti number $\beta_{i, j}$ is the dimension of the $i$ th cohomology of this complex; the dimensions of cohomology of such complexes are upper semicontinuous.

Remark 2.7. Graded Betti numbers of flat families of ideal sheaves on projective space are not semicontinuous. For example, let three points in $\mathbb{P}^{2}$ move from linearly independent position for $u \neq 0$ to collinear position when $u=0$. For $u \neq 0$, the ideal sheaf $\tilde{I}_{u}$ is generated by three quadrics having two linear syzygies. At $u=0$, the ideal sheaf $\tilde{I}_{0}$ is a complete intersection of type $(1,3)$ (with one linear generator, one cubic generator, and just one syzygy).

The point is that the sheaf $\tilde{I}_{0}$ is the sheafification of the flat limit ideal $I_{0}$. In the previous example, the flat limit ideal has an embedded point at the origin, which is lost in the sheafification.

Our brief proof does not recover the "consecutive cancellation" as in [41], but we will not use consecutive cancellation.

\subsection{Gorenstein Artin Algebras}

Let $A$ be an algebra. Most of the time, we will consider standard graded algebras, that is, $A$ is a graded algebra with $A_{0}=\mathbb{C}$, and $A$ is generated in degree 1 . In this situation, the embedding dimension of $A$ is $\operatorname{dim} A_{1}$. Let $\mathfrak{m}=\bigoplus_{i>0} A_{i}$ be the graded maximal ideal. The socle of a graded algebra $A$ is the ideal $\operatorname{Soc}(A)=$ $(0: \mathfrak{m})$, that is, the annihilator of the graded maximal ideal in $A$. When $A$ is Artinian, the socle includes $A_{d}$ where $d=\max \left\{i: A_{i} \neq 0\right\}$. When $A$ is Artinian, $A$ is Gorenstein if and only if $\operatorname{Soc}(A)$ is one-dimensional. The socle degree of $A$ is $\max \left\{i: A_{i} \neq 0\right\}$.

We use [18, Cor. 21.16]. Let $F$ be a concise homogeneous form of degree $d$ in $n$ variables, and $I=F^{\perp}$ be a zero-dimensional Gorenstein ideal, so that $A=T / I$ is a Gorenstein Artin algebra. Then $A$ has socle degree $d=\operatorname{deg} F$ and embedding 
dimension $n$. Let $A=T / I$ have the minimal free resolution $M_{\bullet}$ :

$$
0 \leftarrow T=M_{0} \stackrel{d_{1}}{\leftarrow} M_{1} \stackrel{d_{2}}{\leftarrow} \ldots \stackrel{d_{n}}{\leftarrow} M_{n} \leftarrow 0 .
$$

The resolution $M_{\bullet}$ is self-dual, that is, isomorphic to its dual, up to shifts in grading and homological degrees. We call this isomorphism the Gorenstein symmetry. In particular, writing each $M_{i}=\bigoplus_{j} T\left(-a_{j}^{i}\right)$, we have:

$$
M_{n}=M_{0}^{*}=T(-d-n) \quad \text { and } \quad M_{n-i}=M_{i}^{*}=\bigoplus T\left(-d-n+a_{j}^{i}\right) .
$$

The main focus of this paper is Gorenstein ideals having a minimal generator in degree $d$, that is $\beta_{1, d}(I)>0$. Throughout the article, we will frequently use the following consequence of the Gorenstein symmetry:

$$
\beta_{1, d}\left(F^{\perp}\right)=\beta_{n-1, n}\left(F^{\perp}\right), \quad \text { thus } \beta_{1, d}\left(F^{\perp}\right)>0 \Longleftrightarrow \beta_{n-1, n}\left(F^{\perp}\right)>0 .
$$

As we shall see, $\beta_{n-1, n}\left(F^{\perp}\right)$ can be easier to control than $\beta_{1, d}\left(F^{\perp}\right)$.

We will also use the more elementary symmetry of the Hilbert function of a graded Gorenstein Artin algebra, $h_{A}(i)=h_{A}(d-i)$ for a Gorenstein Artin algebra $A$ of socle degree $d$. See, for example, [52, Thm. 4.1].

We will make use of the following two results. The first is a special case of [19, Thm. 8.18].

Lemma 2.8 ([19, Thm. 8.18]). Suppose that $I \subset T$ is a homogeneous ideal with $\beta_{n-1, n}(I)>0$ and no linear generators. Then there exists a choice of coordinates $\alpha_{1}, \ldots, \alpha_{n}$ of $T$ and linearly independent linear forms $\ell_{1}, \ldots, \ell_{k} \in T_{1}$ for some $0<k<n$ such that the $2 \times 2$ minors of the following matrix are contained in $I$ :

$$
\left(\begin{array}{cccccc}
\alpha_{1} & \cdots & \alpha_{k} & \alpha_{k+1} & \cdots & \alpha_{n} \\
\ell_{1} & \cdots & \ell_{k} & 0 & \cdots & 0
\end{array}\right) .
$$

The second is a special case of [19, Thm. 8.11].

Lemma 2.9 ([19, Thm. 8.11]). Suppose that $n \geq 2, I \subset T$ is a homogeneous ideal containing no linear forms, and $J \subset I$ is a homogeneous subideal. Then $\beta_{n-1, n}(J) \leq \beta_{n-1, n}(I)$.

In Section 5, we will also mention Gorenstein Artin algebras that are not necessarily graded. More precisely, we will consider finite Gorenstein schemes that are spectra of those algebras. These schemes arise naturally when treating deformations of graded Gorenstein Artin algebras.

\subsection{Connected Sum}

When $A, A^{\prime}$ are graded Gorenstein Artin algebras over a field $\mathbb{k}$, both of socle degree $d$, the (formal) connected sum $A \# A^{\prime}$ is defined as follows [37; 51]. $A \# A^{\prime}$ 
is the graded algebra with graded pieces

$$
\left(A \# A^{\prime}\right)_{k}= \begin{cases}\mathbb{k}, & k=0, \\ A_{k} \oplus A_{k}^{\prime}, & 0<k<d, \\ \mathbb{k} & k=d,\end{cases}
$$

in which the products of two elements in $A$ or in $A^{\prime}$ are as before modulo the identification of $A_{d} \cong A_{d}^{\prime} \cong\left(A \# A^{\prime}\right)_{d}$, and the product of a positive-degree element in $A$ with one in $A^{\prime}$ is zero. (See also [1;2] for more general constructions.)

The name the "connected sum" of algebras is motivated by the following example. If $X, Y$ are $d$-dimensional connected closed manifolds with cohomology rings $A_{X}, A_{Y}$, then the cohomology ring of the connected sum $X \# Y$ is the connected sum of the cohomology rings: $A_{X \# Y}=A_{X} \# A_{Y}$.

When a polynomial is a direct sum as we have defined it, its apolar algebra is a connected sum in the stated sense (see also [33, Lemma 3.27]).

Proposition 2.10. If $F=G-H$ is a direct sum decomposition, then $A_{F}=$ $A_{G} \# A_{H}$.

Proof. Let $d=\operatorname{deg} F, T, T^{\alpha}$, and $T^{\beta}$ be as in the proof of Lemma 1.12.

By Lemma 1.12 the annihilators satisfy $\left(G^{\perp}\right)_{k} \cap\left(H^{\perp}\right)_{k}=\left(F^{\perp}\right)_{k}$ when $k<d$. Note that $T_{1}^{\alpha} \subset H^{\perp}$ and $T_{1}^{\beta} \subset G^{\perp}$. Thus for $p, q>0, T_{p}^{\alpha} T_{q}^{\beta} \subset G^{\perp} \cap H^{\perp} \subset F^{\perp}$. Recall that $G^{\perp}$ is the apolar ideal of $G$ in $T$, that is, of $G$ as an element of $S$; the apolar ideal of $G$ in $T^{\alpha}$ (considering $G \in S^{x}$ ) is $G^{\perp} \cap T^{\alpha}$, and similarly for $H$. Hence, for $0<k<d$,

$$
\begin{aligned}
\left(A_{F}\right)_{k} & =T_{k} / F_{k}^{\perp}=\left(\bigoplus_{p+q=k} T_{p}^{\alpha} T_{q}^{\beta}\right) / F^{\perp} \\
& =\left(T^{\alpha}\right)_{k} /\left(G^{\perp} \cap T^{\alpha}\right)_{k} \oplus\left(T^{\beta}\right)_{k} /\left(H^{\perp} \cap T^{\beta}\right)_{k}=\left(A_{G}\right)_{k} \oplus\left(A_{H}\right)_{k},
\end{aligned}
$$

as claimed.

We can use this to give a simple "toy" application of our results. Suppose that $X$ and $Y$ are $d$-dimensional connected closed complex manifolds with cohomology rings $A_{X}$ and $A_{Y}$, and suppose that these rings are standard graded (which is by no means typical: cohomology rings of manifolds can contain generators of different degrees). Write $A_{X}=S^{x} / G^{\perp}$ and $A_{Y}=S^{y} / H^{\perp}$. Then the connected sum $X \# Y$ has cohomology ring $A_{X \# Y} \cong A_{X} \# A_{Y} \cong\left(S^{x} \otimes S^{y}\right) /(G+H)^{\perp}$. Therefore, if $M$ is a $d$-dimensional connected closed complex manifold whose cohomology ring $A_{M}=S / F^{\perp}$ is standard graded and $F$ is not decomposable as a direct sum, then $M$ is not decomposable as a connected sum, at least not into factors whose cohomology rings are standard graded. In particular, if $F^{\perp}$ has no minimal generator in degree $d$, then this holds by Theorem 1.1.

There are well-known topological consequences of a direct sum decomposition, for example, involving monodromy [45] and logarithmic vector fields [55]. It is not immediately obvious what geometric consequences may follow from a 
direct sum decomposition. R. Lazarsfeld shared with the fourth author the observation that if $F=F_{1}+F_{2}$ is a direct sum, then $\operatorname{Sing}(V(F))=\operatorname{Sing}\left(V\left(F_{1}\right)\right) \cap$ $\operatorname{Sing}\left(V\left(F_{2}\right)\right)$, that is, the singular locus of $F$ is an intersection of two cones with disjoint vertices. Furthermore, defining $\Sigma_{a}(G)=\left\{p \mid \operatorname{mult}_{p}(G)>a\right\}$, the common zero locus of the $a$ th partial derivatives of $G$ (so that $\Sigma_{0}(G)=V(G)$, $\left.\Sigma_{1}(G)=\operatorname{Sing} V(G)\right)$, we have $\Sigma_{a}(F)=\Sigma_{a}\left(F_{1}\right) \cap \Sigma_{a}\left(F_{2}\right)$ for all $a>0$.

One necessary condition for $F$ to be a direct sum can be deduced immediately from Proposition 4.2 of [51], which we state here for the reader's convenience. We use the following terminology, taken from [51]: A standard graded Poincaré duality algebra of formal dimension $d$ is precisely a (standard graded) Gorenstein Artin algebra of socle degree $d$ (together with a choice of a nonzero socle element, which we ignore). The rank of such an algebra $H$ is the dimension of $H_{1}$. The $\times$-length of a subspace $V \subset H_{1}$ is the least integer $c$ such that any product of $c+1$ elements of $V$ is zero in $H$ if such an integer exists; otherwise, the $\times$-length of $V$ is infinite. In particular, $V$ has $\times$-length strictly less than $d$ if and only if any product of $d$ elements of $V$ is zero in $H$.

Proposition 2.11 (Proposition 4.2 of [51]). Let $H$ be a standard graded Poincaré duality algebra of formal dimension $d$. Suppose that there is a codimension one subspace $V \subset H_{1}$ of $\times$-length strictly less than $d$. Then, either

(i) $H$ is indecomposable with respect to the connected sum operation \#, or

(ii) $H$ has rank two, and $H \cong \mathbb{F}[x, y] /\left(x y, x^{d}-y^{d}\right) \cong\left(\mathbb{F}[x] /\left(x^{d+1}\right)\right) \#(\mathbb{F}[y] /$ $\left.\left(y^{d+1}\right)\right)$.

See [51] for a proof, valid over any field $\mathbb{F}$. Note that we work over the base field $\mathbb{F}=\mathbb{C}$.

From Proposition 2.11 we can deduce the following necessary condition for $F$ to be a direct sum.

Proposition 2.12. If $F$ has a linear factor, then either $F$ is not a direct sum, or $F=x^{d}-y^{d}$ for some linear forms $x, y$.

Proof. By changing coordinates if necessary, suppose $x_{1}$ divides $F$. Let $W=$ $x_{1}^{\perp}=\left\langle\alpha_{2}, \ldots, \alpha_{n}\right\rangle \subset V^{*}=T_{1}$. This is a codimension 1 subspace whose $d$ th power is in $F^{\perp}$, that is, $w_{1} \cdots w_{d} \in F^{\perp}$ for every $w_{1}, \ldots, w_{d} \in W$. Indeed, each monomial appearing in $F$ has at least one factor $x_{1}$ and hence is annihilated by every product of $d$ elements of $W$.

Let $H=A_{F}=T / F^{\perp}$. Then $W_{1}=W / F_{1}^{\perp}$ is a codimension 1 subspace of $H_{1}$ such that the product of any $d$ elements in $W_{1}$ is zero in $H$. By Proposition 2.11, either $H$ is indecomposable with respect to the connected sum operation, or $H \cong$ $\mathbb{C}[\alpha, \beta] /\left(\alpha \beta, \alpha^{d}-\beta^{d}\right) \cong\left(\mathbb{C}[\alpha] / \alpha^{d+1}\right) \#\left(\mathbb{C}[\beta] / \beta^{d+1}\right)$. In the first case, it follows that $F$ is not a direct sum. In the second case, it follows that $F=x^{d}-y^{d}$ after a suitable change of coordinates. 
We remark in passing that this proposition is essentially just a restatement of Proposition 2.11. We have seen that if $F$ has a linear factor, then there is a codimension 1 subspace $W_{1} \subset\left(A_{F}\right)_{1}$ such that the product of any $d$ elements in $W_{1}$ is zero in $A_{F}$, that is, the $\times$-length of $W_{1}$ is less than $d$. Conversely, if $W_{1}$ is such a subspace, say $W_{1}=x_{1}^{\perp}=\left\langle\alpha_{2}, \ldots, \alpha_{n}\right\rangle$, then every degree $d$ monomial in $\alpha_{2}, \ldots, \alpha_{n}$ annihilates $F$, so $F$ does not contain any terms that are monomials in just the variables $x_{2}, \ldots, x_{n}$. That is, every monomial appearing in $F$ has at least one factor $x_{1}$, and so $F$ is divisible by $x_{1}$. Thus, the hypotheses of Proposition 2.11 and Proposition 2.12 are equivalent. Similarly, the conclusions are equivalent.

\section{Dimension of Direct Sum Locus and Uniqueness}

We discuss the uniqueness of the subspaces over which $F \in$ DirSum splits, and we compute the dimension of DirSum.

\subsection{Uniqueness of Direct Sum Decompositions}

Thom [54] conjectured that every germ at 0 of an analytic function $F$ has a unique finest decomposition as a sum of germs of functions in independent variables, up to analytic equivalence. This means that if

$$
F=F_{1}+F_{2}+\cdots+F_{k}
$$

with $F_{i}$ in independent variables and each $F_{i}$ cannot be written as such a sum, then Thom expected that for any other such decomposition $F=G_{1}+G_{2}+\cdots+G_{l}$, we must have $k=l$, and there exists an analytic isomorphism near 0 preserving $F$ and transporting $G_{i}$ to $F_{i}$ (up to permuting the $G_{i}$ ). This was proved for quasi-homogeneous functions in [22]. We may ask also if a homogeneous polynomial has a unique finest decomposition as a sum of polynomials in independent variables.

More generally, for a homogeneous polynomial $F$, we say that one direct decomposition is finer than another if every direct summand subspace appearing in the second decomposition is a direct sum of subspaces appearing in the first (finer) one. That is, if $F=G_{1}+\cdots+G_{k}$ with $G_{i} \in S^{d} V_{i}$ for $i=1, \ldots, k$ and $V_{1} \oplus \cdots \oplus V_{k}=V$ and also $F=G_{1}^{\prime}+\cdots+G_{l}^{\prime}$ with $G_{j}^{\prime} \in S^{d} V_{j}^{\prime}$ for $j=1, \ldots, l$ and $V_{1}^{\prime} \oplus \cdots \oplus V_{l}^{\prime}=V$, then the direct sum decomposition $G_{1}+\cdots+G_{l}$ is finer than $G_{1}^{\prime}+\cdots+G_{k}^{\prime}$ if every $V_{i}^{\prime}$ is a direct sum of one or more of the $V_{j}$.

Clearly, if $F$ is concise, then a direct sum decomposition $F=G_{1}+\cdots+$ $G_{k}$ is maximally fine if and only if each summand $G_{i} \in S^{d} V_{i}$ is concise with respect to $V_{i}$ and indecomposable as a direct sum. Clearly, every concise $F$ has a maximally fine direct sum decomposition. The uniqueness question asks whether every concise $F$ has a unique maximally fine direct sum decomposition.

In fact, quadrics decompose as direct sums over many splittings of the vector space: for example, $x^{2}+y^{2}=(c x+s y)^{2}+(s x-c y)^{2}$ for any $c, s$ such that $c^{2}+s^{2}=1$. For this reason, we usually restrict to degrees $d \geq 3$ and sometimes to $d \geq 4$. In these degrees, the question of uniqueness has a positive answer. 
Theorem 3.1 ([33, Thm. 3.7]). Let $F$ be a concise form of degree $d \geq 3$. Then $F$ has a unique maximally fine direct sum decomposition.

In fact, [33, Thm. 3.7] holds in any characteristic and gives a description of the subspaces appearing in the maximally fine direct sum decomposition. Moreover, [50, Prop. 3.1] provides an analogous uniqueness decomposition for connected sums of Gorenstein Artin algebras. However, the proof of Theorem 3.1 requires some preparation, which lies outside the scope of this paper.

Here we show a weaker statement: essentially that the direct sum decomposition is uniquely determined for forms in an open dense subset of DirSum. This is sufficient for our purposes and does not require many tools other than those already introduced.

It is easy for binary forms.

Proposition 3.2. Every direct sum in two variables of degree $d \geq 3$ has a uniquely determined decomposition.

Proof. There is a unique (up to scalar) generator of the apolar ideal in degree 2 (and another in degree $d$ ). Writing $F=x^{d}-y^{d}$, this quadratic apolar generator is $Q=\alpha \beta$, and the pair of subspaces $\langle x\rangle,\langle y\rangle$ over which $F$ decomposes is determined as the pair of lines corresponding to the pair of points in projective space $\{[x],[y]\}=V(Q)$.

To go further, we use the notion of compressed algebras; see Definition 3.11 and Proposition 3.12 of [29]. We recall not the most general definition, but just the definition in the case that $A=A_{F}=T / F^{\perp}$ is a graded Gorenstein Artin algebra of socle degree $d$. In this case, $A$ is compressed if, for each $i \in\{0,1, \ldots, d\}$, we have $\operatorname{dim} A_{i}=\min \left(\operatorname{dim} S^{i}\left(A_{1}\right), \operatorname{dim} S^{d-i}\left(A_{1}\right)\right)$. If we have chosen $T$ and the isomorphism $A=A_{F}=T / F^{\perp}$ in such a way that $F$ is concise, then $A$ is compressed if and only if $\operatorname{dim} A_{i}=\min \left(\operatorname{dim} T_{i}, \operatorname{dim} T_{d-i}\right)$. When $F \in S^{d} V$ is general, $A_{F}$ is compressed [29, Prop. 3.12]. (Recall that a general element of a variety is any element of a suitable dense open subset of the variety.)

Lemma 3.3. Let $d \geq 4$ and $n=\operatorname{dim} V \geq 2$. For $F \in S^{d} V$ general, $F^{\perp}$ has no quadratic generators, and $F$ is not decomposable as a direct sum.

Proof. We have that $A_{F}$ is compressed. This implies $F^{\perp}$ has no generators in degrees less than or equal to $d / 2 \operatorname{since} \operatorname{dim}\left(F^{\perp}\right)_{i}=\operatorname{dim} T_{i}-\operatorname{dim} A_{i}=0$ for $0 \leq$ $i \leq d / 2$. In particular, $\left(F^{\perp}\right)_{2}=0$, so that $F$ is not decomposable as a direct sum (see Corollary 1.14).

Table 1 shows that a general cubic in $n=3$ variables is not decomposable as a direct sum. But a general binary cubic is decomposable as a direct sum: Let $F=F(x, y)$ be a binary cubic with distinct roots. By a linear substitution we may move those roots to be the cubic roots of unity; in these coordinates, $F=x^{3}-y^{3}$. 
Proposition 3.4. For $d \geq 4$ and $n \geq 2$, there is a dense subset of DirSum (that is, a union of a dense subset of each irreducible component of DirSum) such that for $F$ in this subset, $F$ decomposes as a direct sum over a uniquely determined pair of subspaces.

Proof. Let $F \in$ DirSum be arbitrary, and let $F=G+H$ with $G \in S^{d} V_{1}, H \in$ $S^{d} V_{2}, V_{1} \oplus V_{2}=V$. Now, let $G^{\prime} \in S^{d} V_{1}, H^{\prime} \in S^{d} V_{2}$ be general, and $F^{\prime}=G^{\prime}+$ $H^{\prime}$. As $G^{\prime} \rightarrow G$ and $H^{\prime} \rightarrow H$, we have $F^{\prime} \rightarrow F$. Clearly, $F^{\prime}$ decomposes as a direct sum over $V_{1} \oplus V_{2}$; we claim that this is the unique pair of subspaces over which $F^{\prime}$ decomposes. We have $\left(F^{\prime \perp}\right)_{2}=\left(G^{\prime \perp}\right)_{2} \cap\left({H^{\prime}}^{\perp}\right)_{2}$; see Lemma 1.12. Since $A_{G^{\prime}}$ and $A_{H^{\prime}}$ are compressed, $G^{\prime \perp}$ and $H^{\prime \perp}$ have no quadratic generators other than $\left(G^{\prime \perp}\right)_{2}=V_{2}^{*} V^{*}$ and $\left(H^{\prime \perp}\right)_{2}=V^{*} V_{1}^{*}$. In particular, then $\left(F^{\prime \perp}\right)_{2}=$ $V_{1}^{*} V^{*} \cap V^{*} V_{2}^{*}=V_{1}^{*} V_{2}^{*}$, and $V_{1} \cup V_{2}$ is the zero locus $V\left(\left(F^{\prime \perp}\right)_{2}\right)$. Hence, $V_{1}$ and $V_{2}$ are uniquely determined by $F^{\prime}$, as claimed. This shows that there is a dense subset of DirSum whose elements decompose as direct sums over uniquely determined pairs of subspaces.

On the other hand, there exists an open dense subset of cubic direct sums in three variables for which the decomposition as a direct sum over two subspaces is not unique. Indeed, a cubic direct sum in three variables can be written as $F=x^{3}+G(y, z)$. If $G$ is a general cubic binary form, then (with another change of coordinates) $F=x^{3}+y^{3}+z^{3}$. However, we do see that $F$ decomposes as a direct sum over $V=\langle x\rangle \oplus\langle y\rangle \oplus\langle z\rangle$, and this finest decomposition is uniquely determined by $F$, as $\{[x],[y],[z]\}=V(\alpha \beta, \alpha \gamma, \beta \gamma)=V\left(\left(F^{\perp}\right)_{2}\right)$.

\subsection{Dimension}

For $V_{1} \oplus V_{2}=V$ let $\operatorname{DirSum}^{*}\left(V_{1}, V_{2}\right)=S^{d}\left(V_{1}\right) \oplus S^{d}\left(V_{2}\right)$; note that this contains degenerate sums involving $0 \in S^{d}\left(V_{1}\right)$ or $0 \in S^{d}\left(V_{2}\right)$. For $a+b=n, a \leq b$, let $\operatorname{DirSum}^{*}(a, b)$ be the union of the $\operatorname{DirSum} *\left(V_{1}, V_{2}\right)$ for $\operatorname{dim} V_{1}=a, \operatorname{dim} V_{2}=b$. Each DirSum* $(a, b)$ is irreducible since it is the image of the natural projection

$$
\begin{aligned}
S^{d}\left(\mathbb{C}^{a}\right) \times S^{d}\left(\mathbb{C}^{b}\right) \times \mathrm{GL}_{n}(\mathbb{C}) & \rightarrow S^{d}(V), \\
(G, H, M) & \mapsto G\left(m_{1}, \ldots, m_{a}\right)+H\left(m_{a+1}, \ldots, m_{n}\right),
\end{aligned}
$$

where the $m_{i}$ are the columns of the matrix $M$. Of course, this is not injective.

For each $a+b=n$, let $\operatorname{DirSum}(a, b) \subset \operatorname{DirSum}^{*}(a, b)$ be the subset of $F$ which are indeed decomposable as direct sums in which one term involves $a$ variables and the other involves $b$ variables, that is, discarding those elements of $\operatorname{DirSum}^{*}(a, b)$ in which one or both terms are identically zero. Further, let $\operatorname{DirSum}^{\circ}(a, b) \subset \operatorname{DirSum}(a, b)$ be the subset of concise forms $F$. Then $\operatorname{DirSum}^{\circ}(a, b)$ is a Zariski open subset of $\operatorname{DirSum}^{*}(a, b)$, since its complement is defined by rank conditions on the catalecticant $C_{F}^{1}$.

Now DirSum $=\bigcup_{a+b=n} \operatorname{DirSum}(a, b)$. We see that DirSum contains the dense subset $\operatorname{DirSum}^{\circ}=\bigcup_{a+b=n} \operatorname{DirSum}^{\circ}(a, b)$, that is, a union of a dense open subset of each $\operatorname{DirSum}(a, b)$. 
Proposition 3.5. For $d \geq 4$ and $n \geq 3, \operatorname{dim}_{\operatorname{DirSum}}^{*}(a, b)=2 a b+\left(\begin{array}{c}d+a-1 \\ a-1\end{array}\right)+$ $\left(\begin{array}{c}d+b-1 \\ b-1\end{array}\right)$ and $\operatorname{dim} \operatorname{DirSum}=2(n-1)+1+\left(\begin{array}{c}d+n-2 \\ n-2\end{array}\right)$.

Proof. Let $\operatorname{DirSum}^{\circ \circ}(a, b) \subset \operatorname{DirSum}^{\circ}(a, b)$ be the set of $F=G+H$ such that $A_{G}$ and $A_{H}$ are compressed. There is a map from $\operatorname{DirSum}^{\circ \circ}(a, b)$ to $G(a, V) \times G(b, V)$ whose general fiber has dimension $\left(\begin{array}{c}d+a-1 \\ a-1\end{array}\right)+\left(\begin{array}{c}d+b-1 \\ b-1\end{array}\right)$. This shows $\operatorname{dim} \operatorname{DirSum}^{*}(a, b)$ is as claimed. This dimension is maximized when $(a, b)=(1, n-1)$.

A more refined dimension formula is found in [33, Thm. 3.47]. Moreover, an analogous formula for connected sum Gorenstein algebras is in [51, Prop. 4.4].

\section{Apolar Generators and Limits of Direct Sums}

Let $F \in S^{d} V$ be a homogeneous polynomial of degree $d$. Recall that an equipotent generator of $F$ is a minimal generator of the ideal $F^{\perp}$ of degree $d$. In this section, we collect results that relate quadratic generators to direct sums and to equipotent apolar generators. Then we relate equipotent apolar generators to limits of direct sums.

\subsection{Quadratic Generators}

Forms with an equipotent apolar generator have similar characteristics to forms which are direct sums. Perhaps the best illustration of this is the behavior of quadratic apolar generators.

We make first the following easy observation:

Proposition 4.1. If $F$ is a concise direct sum in $n$ variables, then $F$ has at least $n-1$ quadratic apolar generators.

It was previously shown by Meyer and Smith [37, Lem. VI.2.1] that $F$ has at least one quadratic apolar generator, without assuming $F$ to be concise. Moreover, [33, Thm. 3.35] provides a calculation of all graded Betti numbers of $F^{\perp}$.

Proof of Proposition 4.1. Say $F \in S^{d} V$ is a direct sum over $V=V_{1} \oplus V_{2}$ with $\operatorname{dim} V_{i}=v_{i}, v_{1}+v_{2}=\operatorname{dim} V=n$. Then by Corollary 1.14 we have $V_{1}^{*} V_{2}^{*} \subset$ $\left(F^{\perp}\right)_{2}$, a subspace of dimension $v_{1} v_{2} \geq n-1$. By hypothesis there are no linear forms in $F^{\perp}$, so that everything in $\left(F^{\perp}\right)_{2}$ is a minimal generator.

Conversely, if $V=V_{1} \oplus V_{2}$ and $V_{1}^{*} V_{2}^{*} \subset F^{\perp}$, then $F=F_{1}+F_{2}$ where $F_{1} \in S^{d} V_{1}$, $F_{2} \in S^{d} V_{2}$; see Corollary 1.14. If, furthermore, $F$ is concise, then $F_{1}, F_{2} \neq 0$, and $F$ is a direct sum.

More generally, if $V=V_{1} \oplus \cdots \oplus V_{s}$, then $F=F_{1}+\cdots+F_{s}$ where $F_{i} \in S^{d} V_{i}$ if and only if $\bigoplus_{i<j} V_{i}^{*} V_{j}^{*} \subset F^{\perp}$ as quadratic generators, where $V_{i}^{*}=\bigcap_{j \neq i} V_{j}^{\perp}$. (In coordinates, if each $V_{i}$ has a basis $x_{i, 1}, \ldots, x_{i, n_{i}}$, then $V_{i}^{*}$ is spanned by the dual basis elements $\alpha_{i, 1}, \ldots, \alpha_{i, n_{i}}$.) If this holds, and furthermore $F$ is concise, then each $F_{i} \neq 0$, and $F$ is an $s$-fold direct sum. 
COROLlARY 4.2. If $F$ is a concise form in $n$ variables which is an $s$-fold direct sum for $s \geq 2$ then $F^{\perp}$ has at least $(s-1)(2 n-s) / 2$ quadratic generators.

Proof. When $F$ is a direct sum over $V=V_{1} \oplus \cdots \oplus V_{s}, F^{\perp}$ contains $\bigoplus_{i<j} V_{i}^{*} V_{j}^{*}$ as quadratic generators; see Corollary 1.14. The fewest quadratic generators arise when the summands $V_{1}, \ldots, V_{s}$ have dimensions $1, \ldots, 1, n+1-s$, yielding the statement.

Less obviously we have the following:

Proposition 4.3. If $F$ is a concise form in $n$ variables and $F$ has an equipotent apolar generator, then $F$ has at least $n-1$ quadratic apolar generators.

Proof. Since $F$ is concise, $F^{\perp}$ has no linear generators. Then the quadratic elements provided by Lemma 2.8 are minimal generators, and there are at least $n-1$ independent ones, for example, the $2 \times 2$ minors given by the first and $i$ th columns of (3) for $2 \leq i \leq n$.

Note that the Fermat hypersurface $x_{1}^{d}+\cdots+x_{n}^{d}$ has $\left(\begin{array}{c}n \\ 2\end{array}\right)$ quadratic apolar generators. This is the maximum number possible for smooth forms, as the following easy observation shows.

Proposition 4.4. If $F$ defines a smooth hypersurface of degree $d \geq 3$ on $\mathbb{P}^{n-1}$, then $F^{\perp}$ has at most $\left(\begin{array}{l}n \\ 2\end{array}\right)$ quadratic generators. More generally, if the set of points in $\mathbb{P}^{n-1}$ at which $F$ vanishes to order $\geq$ a has dimension $k$, then $\operatorname{dim}\left(F^{\perp}\right)_{d-a+1} \leq$ $\left(\begin{array}{l}n+d-a \\ d-a+1\end{array}\right)-n+k+1$.

Proof. Otherwise, $\mathbb{P} F_{d-a+1}^{\perp} \subset \mathbb{P} T_{d-a+1}$ necessarily has a $(k+1)$-dimensional intersection with the Veronese variety $v_{d-a+1}\left(\mathbb{P} T_{1}\right)$, since it has codimension $\left(\begin{array}{l}n+d-a \\ d-a+1\end{array}\right)-n$. For each $\left[\alpha^{d-a+1}\right]$ in this intersection, $F$ vanishes to order at least $a$ at $[\alpha]$ by Lemma 2.2. This gives a $(k+1)$-dimensional set along which $F$ vanishes to order $a$. The first statement follows with $a=d-1 \geq 2$ and $k=-1$ when $V(F)$ is smooth.

Having the maximum number of quadratic apolar generators does not characterize Fermat hypersurfaces, however; the concise plane cubics all have the maximum number of quadratic apolar generators; see Table 1.

\subsection{Equipotent Apolar Generators and Limits of Direct Sums}

Fix a degree $d$ and number of variables $n$. Let $V$ be a vector space with $\operatorname{dim} V=n$. In this section, we first prove that if $F \in S^{d} V$ has an equipotent apolar generator, then $F$ is a limit of direct sums. We next prove that if $F$ is also concise, then the converse holds. This assumption is needed by Example 1.5.

THEOREM 4.5. If $F$ has an equipotent apolar generator, then $F$ is a limit of direct sums. Moreover, either $F$ is a direct sum, or it can be written in the following 
normal form, for some choice of basis $x_{1}, \ldots, x_{k}, y_{1}, \ldots, y_{k}, z_{1}, \ldots, z_{n-2 k}$ of $V$ :

$$
F(x, y, z)=\sum x_{i} \frac{\partial H(y)}{\partial y_{i}}+G(y, z) .
$$

Here $G \in S^{d}\left\langle y_{1}, \ldots, y_{k}, z_{1}, \ldots, z_{n-2 k}\right\rangle$ and $H \in S^{d}\left\langle y_{1}, \ldots, y_{k}\right\rangle$.

Proof. We immediately reduce to the case that $F$ is concise: If $F$ is concise over $W \subset V$, then we will write $F$ as a limit of direct sums which are in $S^{d} W$.

We assume that $F^{\perp}$ has a generator in degree $d=\operatorname{deg} F$. By Gorenstein symmetry (2), $\beta_{n-1, n}\left(F^{\perp}\right)>0$. By Lemma 2.8 there are linearly independent linear forms $\ell_{1}, \ldots, \ell_{k}$ for some $0<k<n$ such that $F^{\perp}$ contains the $2 \times 2$ minors of the matrix

$$
\left(\begin{array}{cccccc}
\alpha_{1} & \cdots & \alpha_{k} & \alpha_{k+1} & \cdots & \alpha_{n} \\
\ell_{1} & \cdots & \ell_{k} & 0 & \cdots & 0
\end{array}\right) .
$$

Let $L: T_{1} \rightarrow T_{1}$ be the linear map given by $L\left(\alpha_{i}\right)=\ell_{i}$ for $1 \leq i \leq k, L\left(\alpha_{i}\right)=0$ for $i>k$. That is, for all $i, j, \alpha_{i} L\left(\alpha_{j}\right)-\alpha_{j} L\left(\alpha_{i}\right) \in F^{\perp}$. By linearity, $v L(w)-$ $w L(v) \in F^{\perp}$ for all $v, w \in T_{1}$. Let $\tilde{L}: \wedge^{2} T_{1} \rightarrow F^{\perp}$ be defined by $\tilde{L}(v \wedge w)=$ $v L(w)-w L(v) \in F^{\perp}$.

Since $0<k<n, L$ is not zero or a scalar multiple of the identity and has a nontrivial kernel. We begin by changing basis in $V^{*}$ (and dually in $V$ ) to put $L$ into Jordan normal form. It turns out that if $L$ has distinct eigenvalues, then $F$ decomposes as a direct sum over the generalized eigenspaces of $L$; otherwise, if $L$ is a nonzero nilpotent matrix, then $F$ is a limit of direct sums.

Suppose first that $\lambda_{i} \neq \lambda_{j}$ are distinct eigenvalues of $L$. Then there are some positive integers $v_{i}, v_{j}$ such that $\left(L-\lambda_{i}\right)^{v_{i}} \alpha_{i}=\left(L-\lambda_{j}\right)^{v_{j}} \alpha_{j}=0$ but $\left(L-\lambda_{i}\right)^{v_{i}-1} \alpha_{i},\left(L-\lambda_{j}\right)^{v_{j}-1} \alpha_{j} \neq 0$. We show that $\alpha_{i} \alpha_{j} \in \operatorname{image}(\tilde{L}) \subset F^{\perp}$ by induction on $v_{i}+v_{j}$. The induction begins with $v_{i}=v_{j}=1$. Then $\tilde{L}\left(\alpha_{i} \wedge \alpha_{j}\right)=$ $\alpha_{i} L\left(\alpha_{j}\right)-\alpha_{j} L\left(\alpha_{i}\right)=\left(\lambda_{j}-\lambda_{i}\right) \alpha_{i} \alpha_{j}$. If, say, $v_{i}>1$, so that $L\left(\alpha_{i}\right)=\lambda_{i} \alpha_{i}+\alpha_{i-1}$, then $\tilde{L}\left(\alpha_{i} \wedge \alpha_{j}\right)=\left(\lambda_{j}-\lambda_{i}\right) \alpha_{i} \alpha_{j}+\alpha_{i-1} \alpha_{j} \in F^{\perp}$. Since $\alpha_{i-1} \alpha_{j} \in F^{\perp}$ by induction, $\alpha_{i} \alpha_{j} \in F^{\perp}$.

This shows that, for the generalized eigenspace decomposition $V^{*}=\bigoplus_{\lambda} V_{\lambda}^{*}$, we have $\left(V_{\lambda}^{*}\right)\left(\bigoplus_{\mu \neq \lambda} V_{\mu}^{*}\right) \subset F^{\perp}$ for each eigenvalue $\lambda$. Thus, $F=\sum_{\lambda} F_{\lambda}, F_{\lambda} \in$ $S^{d} V_{\lambda}$ where $V_{\lambda}=\left(V_{\lambda}^{*}\right)^{*}=\bigcap_{\mu \neq \lambda}\left(V_{\mu}^{*}\right)^{\perp}$ by Corollary 1.14 . Since $F$ is concise, each $F_{\lambda}$ must be nonzero (in fact, concise with respect to $V_{\lambda}$ ). In this case, then, $F$ is a direct sum.

Now we see what happens when $L$ has just one eigenvalue. Then $L$ is nilpotent since $\operatorname{ker} L \neq 0$. We claim that image $\left(\widetilde{L^{v}}\right) \subset F^{\perp}$ for all $v \geq 1$. Indeed,

$$
\begin{aligned}
\widetilde{L^{\nu}}(\alpha \wedge \beta) & =\alpha \cdot L^{\nu}(\beta)-\beta \cdot L^{\nu}(\alpha) \\
& =\alpha L\left(L^{\nu-1} \beta\right)-\left(L^{\nu-1} \beta\right)(L \alpha)+(L \alpha)\left(L^{\nu-1} \beta\right)-\beta\left(L^{\nu-1} L \alpha\right) \\
& =\tilde{L}\left(\alpha \wedge L^{\nu-1} \beta\right)+\widetilde{L^{\nu-1}}(L(\alpha) \wedge \beta),
\end{aligned}
$$

which is in $F^{\perp}$ by induction. Now suppose $L^{v+1}=0 \neq L^{v}$; we replace $L$ with $L^{v}$, so we can assume that $L^{2}=0 \neq L$. Say $k=\operatorname{rank} L$. Then the Jordan normal form of $L$ yields a basis $\alpha_{1}, \ldots, \alpha_{k}, \beta_{1}, \ldots, \beta_{k}, \gamma_{1}, \ldots, \gamma_{n-2 k}$ of $V^{*}$ such 
that $L\left(\beta_{i}\right)=\alpha_{i}$ and $L\left(\alpha_{i}\right)=L\left(\gamma_{i}\right)=0$. So $L$ can be written in block form with respect to this basis,

$$
L=\left(\begin{array}{lll}
\alpha & \beta & \gamma \\
0 & I & 0 \\
0 & 0 & 0 \\
0 & 0 & 0
\end{array}\right) \begin{gathered}
\alpha \\
\gamma \\
\gamma
\end{gathered} .
$$

We give $V$ the dual basis $x_{1}, \ldots, x_{k}, y_{1}, \ldots, y_{k}, z_{1}, \ldots, z_{n-2 k}$. Now $\tilde{L}\left(\alpha_{i} \wedge \beta_{j}\right)=$ $\alpha_{i} \alpha_{j} \in F^{\perp}$ and $\tilde{L}\left(\gamma_{i} \wedge \beta_{j}\right)=\gamma_{i} \alpha_{j} \in F^{\perp}$. Since

$$
\left\langle\alpha_{1}, \ldots, \alpha_{k}\right\rangle\left\langle\alpha_{1}, \ldots, \alpha_{k}, \gamma_{1}, \ldots, \gamma_{n-2 k}\right\rangle \subset F^{\perp},
$$

we have $F=\sum x_{i} H_{i}(y)+G(y, z)$ where $\operatorname{deg} H_{i}=d-1, \operatorname{deg} G=d$. Furthermore, $\tilde{L}\left(\beta_{i} \wedge \beta_{j}\right)=\alpha_{j} \beta_{i}-\alpha_{i} \beta_{j} \in F^{\perp}$, so $\partial H_{i} / \partial y_{j}=\partial H_{j} / \partial y_{i}$. Thus, there exists $H(y)$ such that $H_{i}=\partial H / \partial y_{i}$. This shows the normal form part of the statement of the theorem. Finally, we write $F$ as a limit of direct sums as follows:

$$
F=\lim _{t \rightarrow 0} \frac{1}{t}\left(H\left(y_{1}+t x_{1}, \ldots, y_{k}+t x_{k}\right)+t G(y, z)-H(y)\right),
$$

which, for $t \neq 0$, is a direct sum over $\left\langle y_{i}+t x_{i}\right\rangle \oplus\langle y, z\rangle$.

EXAMPLE 4.6. Let $F=x y^{d-1}$, so that $F^{\perp}=\left\langle\alpha^{2}, \beta^{d}\right\rangle$. Then $F^{\perp}$ contains the $2 \times 2$ minors of the matrix

$$
\left(\begin{array}{ll}
\alpha & \beta \\
0 & \alpha
\end{array}\right)
$$

In the notation of the above proof, $L: T_{1} \rightarrow T_{1}$ is given by $L(\alpha)=0, L(\beta)=\alpha$. Then $L$ is nilpotent, $L^{2}=0$. The next step in the proof provides a decomposition $F=x H_{1}(y)$, where $\operatorname{deg} H_{1}=d-1$; so $H_{1}(y)=y^{d-1}$. We have $H_{1}=\partial H / \partial y$ where $H(y)=(1 / d) y^{d}$. In the proof's notation, $G=0$. Of course then

$$
F=x y^{d-1}=\lim _{t \rightarrow 0} \frac{1}{d t}\left((y+t x)^{d}-y^{d}\right) .
$$

ExAmPLE 4.7. Let $F=x^{2} y-y^{2} z$. We saw in Example 1.4 that $F^{\perp}=$ $\left\langle\gamma^{2}, \alpha \gamma, \alpha^{2}+\beta \gamma, \beta^{3}, \alpha \beta^{2}\right\rangle$, so $F$ has two equipotent apolar generators. And $F^{\perp}$ contains the $2 \times 2$ minors of the matrix

$$
\left(\begin{array}{ccc}
\alpha & \beta & \gamma \\
-\gamma & \alpha & 0
\end{array}\right)
$$

In the notation of the proof of Theorem 4.5, the endomorphism corresponding to this matrix is $L: T_{1} \rightarrow T_{1}$, given by $L(\alpha)=-\gamma, L(\beta)=\alpha, L(\gamma)=0$. Note that $L$ is nilpotent, $L^{3}=0$. We replace $L$ with $L^{\prime}=L^{2}$, represented by the matrix

$$
\left(\begin{array}{ccc}
\alpha & \beta & \gamma \\
0 & -\gamma & 0
\end{array}\right)
$$

Again $L^{\prime}$ is nilpotent, $L^{\prime 2}=0$. Although the labeling of variables is different than in the proof, the proof's next step yields $F=z H_{1}(y)+G(x, y)$, where 
$H_{1}(y)=-y^{2}$; apparently, $G(x, y)=x^{2} y$. Then $H(y)=-(1 / 3) y^{3}$. We get

$$
\begin{aligned}
F & =\lim _{t \rightarrow 0} \frac{1}{t}(H(y+t z)+t G(x, y)-H(y)) \\
& =\lim _{t \rightarrow 0} \frac{1}{3 t}\left(-(y+t z)^{3}+t x^{2} y+y^{3}\right),
\end{aligned}
$$

a limit of direct sums over the subspaces $\langle y+t z\rangle \oplus\langle x, y\rangle$ for $t \neq 0$.

Theorem 4.5 has several parallels in [33]. The linear map $L$ in its proof corresponds to one of the matrices in [33, Def. 2.14]. The nilpotent case is covered by [33, Thm. 4.5]. In the case of distinct eigenvalues, the projections onto the distinct eigenspaces give the orthogonal idempotent matrices discussed in [33, Prop. 3.5]. An extended result in this case is given in [33, Thm. 3.7].

Now we prove the converse (cf. [33, Lem. 4.2]).

THEOREM 4.8. If $F$ is a concise limit of $s$-fold direct sums, then $F$ has at least $s-1$ equipotent apolar generators.

Proof. Suppose $F=F_{0}$ is a concise limit of $s$-fold direct sums, $F_{0}=\lim F_{t}$. Let $J$ be the flat limit of the ideals $F_{t}^{\perp}$. We have $J \subset F^{\perp}$ since differentiation varies continuously as the $F_{t}$ varies regularly. Indeed, for $\Theta \in J, \Theta=\lim \Theta_{t}$ for $\Theta_{t} \in F_{t}^{\perp}$, so $\left.\Theta_{t}\right\lrcorner F_{t}=0$ for $t \neq 0$; hence, $\left.\left.\Theta\right\lrcorner F=\lim \Theta_{t}\right\lrcorner F_{t}=0$, so $\Theta \in F^{\perp}$. By Proposition 2.6, upper-semicontinuity of graded Betti numbers, $\beta_{n-1, n}(J) \geq$ $s-1$.

Now there is no general inequality between the graded Betti numbers of an arbitrary homogeneous ideal $I$ and those of a homogeneous subideal $J \subset I$; $\beta_{i, j}(I)>\beta_{i, j}(J), \beta_{i, j}(I)<\beta_{i, j}(J)$, and $\beta_{i, j}(I)=\beta_{i, j}(J)$ all are possible. However, in this simple case, we do have the inequality we are looking for by Lemma 2.9. That is, $\beta_{n-1, n}\left(F^{\perp}\right) \geq \beta_{n-1, n}(J) \geq s-1$, since $F$ is concise, meaning $\left(F^{\perp}\right)_{1}=0$. By Gorenstein symmetry (2) there are at least $s-1$ minimal generators of degree $d$ in $F^{\perp}$.

This completes the proof of Theorem 1.7, which comprises Theorems 4.5 and 4.8.

EXAMPLE 4.9. Let $F$ be a concise plane curve $(n=3)$ of degree $d$ having an equipotent apolar generator. Either $F$ is a direct sum, $F=x^{d}+G(y, z)$, or else $F$ is a limit of direct sums of the form $F=x y^{d-1}+G(y, z)$ by the normal form part of Theorem 4.5. Note that if $G(y, z)$ includes terms $a y^{d-1} z+b y^{d}$, then replacing $x$ with $x+a z+b y$ gives us

$$
F=x y^{d-1}+z^{2} G_{d-2}(y, z),
$$

where $\operatorname{deg} G_{d-2}=d-2$. Conversely, if $F$ is of this form, then $F$ is a limit of direct sums and has an equipotent apolar generator.

Thus, a concise plane curve $F$ is a limit of direct sums and has an equipotent apolar generator if and only if, after a linear change of coordinates, either $F=$ $x^{d}+G(y, z)$ or $F=x y^{d-1}+z^{2} G_{d-2}(y, z)$. 


\subsection{Lower Bound for Degree of Apolar Generators}

Here we prove Theorem 1.9, a lower bound for the maximum degree of the apolar generators of a form $F$ in terms of the degree $d$ of $F$ and the number $n$ of variables: we show that $F^{\perp}$ always has a minimal generator of degree at least $(d+n) / n$.

LEMMA 4.10. Suppose that $I \subset T$ is a homogeneous complete intersection ideal of codimension $n$. Then $I=G^{\perp}$ for some homogeneous $G \in S$. If the minimal generators of $I$ are homogeneous of degrees $\delta_{1}, \ldots, \delta_{n}$, then $\operatorname{deg} G=\delta_{1}+\cdots+$ $\delta_{n}-n$.

Proof. The existence of $G$ follows from [18, Thm. 21.6]. The degree of $G$ is equal to the socle degree of $A_{G} \cong T / I$, which is $\sum_{i=1}^{n}\left(\delta_{i}-1\right)$ by, for example, Exercise 21.16 of [18].

We will deduce Theorem 1.9 from the following slightly stronger proposition.

Proposition 4.11. Let $F$ be a homogeneous form of degree $d$ in $n$ variables. Suppose that $F^{\perp}$ has minimal generators $\Theta_{1}, \ldots, \Theta_{s}$ such that $\operatorname{deg} \Theta_{i}=d_{i}$ for each $i$, and let $d_{1} \leq \cdots \leq d_{s}$. Let $\delta$ be an integer such that the ideal $\left(F^{\perp}\right)_{\leq \delta}$ is $\mathfrak{m}$-primary. Assume that $d_{k}=\delta<d_{k+1}$ or $k=s$ and $\delta=d_{s}$; necessarily, $k \geq n$. Then $d \leq d_{k}+d_{k-1}+\cdots+d_{k-n+1}-n$.

Here $\mathfrak{m}$ is the graded maximal ideal in $T$.

Proof of Proposition 4.11. For each $i=k, k-1, \ldots, k-n+1$, let $\Psi_{i} \in\left(F^{\perp}\right)_{d_{i}}$ be general. Since $\left(F^{\perp}\right)_{\leq d_{k}}$ is $\mathfrak{m}$-primary, $\left(F^{\perp}\right)_{d_{i}}$ is a basepoint-free linear series on $V\left(\Psi_{k}, \ldots, \Psi_{i+1}\right)$ for each $i$. Then by Bertini's theorem [32, Thm. I.6.3] and downward induction on $i$, the ideal $\left(\Psi_{k}, \Psi_{k-1}, \ldots, \Psi_{i}\right)$ is a complete intersection for each $i \geq k-n+1$. In particular, $I=\left\langle\Psi_{k}, \ldots, \Psi_{k-n+1}\right\rangle$ is a complete intersection of codimension $n$. By Lemma 4.10, $I=G^{\perp}$ for a form $G$ of degree $d_{k}+\cdots+d_{k-n+1}-n$. By Lemma 2.1, $\left.F=\Theta\right\lrcorner G$ for some $\Theta \in T$, so that $\operatorname{deg} F \leq \operatorname{deg} G$.

Proof of Theorem 1.9. Let $d_{1} \leq \cdots \leq d_{s}$ be the degrees of the minimal generators of $F^{\perp}$ as in the previous proposition. Then, regardless of the value of $k$, we have $d \leq d_{k}+\cdots+d_{k-n+1}-n \leq n \delta-n$.

Suppose that $F$ is a concise homogeneous polynomial in $n$ variables for which $\delta=2$, that is, $F^{\perp}$ is generated by quadrics. Then Theorem 1.9 implies $d=$ $\operatorname{deg} F \leq n$. Moreover, the proof of the theorem shows that $F=\Theta\lrcorner G$ for some $\Theta \in T$ and $G \in S$ such that $\operatorname{deg} G=n$ and $G^{\perp}$ is a complete intersection of $n$ quadrics. For example, let $F$ be a determinant of a generic $k \times k$ matrix,

$$
F=\operatorname{det}\left(\begin{array}{ccc}
x_{11} & \cdots & x_{1 k} \\
\vdots & & \vdots \\
x_{k 1} & \cdots & x_{k k}
\end{array}\right)
$$


In this case, $\operatorname{deg} F=k$, and the number of variables is $k^{2}$. Then as $G$ we may take the monomial $\prod_{i, j=1}^{k} x_{i j}$.

However, it is not true that every homogeneous polynomial of the form $\Theta\lrcorner G$ must have $(\Theta\lrcorner G)^{\perp}$ generated by quadrics. For example, let $G=x_{1} \cdots x_{6}$, let $\Theta=\alpha_{1} \alpha_{2} \alpha_{3}-\alpha_{4} \alpha_{5} \alpha_{6}$, and let $\left.F=\Theta\right\lrcorner G=x_{4} x_{5} x_{6}-x_{1} x_{2} x_{3}$. Then $G^{\perp}$ is a complete intersection of quadrics, but $F^{\perp}$ has a minimal generator of degree 3 by Theorem 1.1, namely, $\alpha_{4} \alpha_{5} \alpha_{6}+\alpha_{1} \alpha_{2} \alpha_{3}$.

The problem of classification of all homogeneous polynomials $F$ with $F^{\perp}$ generated by quadrics appears to be difficult. We expect that the answer must be complex: if $F$ is the permanent of a generic symmetric matrix, then $F^{\perp}$ has minimal generators of degree 3 , whereas the apolar ideal of the determinant of the same matrix has only quadratic generators; see [48, Thm. 3.23, Thm. 3.11]. However, this discussion shows that it might be helpful to first classify $G$ with $d=\operatorname{deg} G=n$ and $G^{\perp}$ generated by quadrics.

Even the classification of $G$ is difficult. For $d=n=2$, any rank two quadric $G$ has $G^{\perp}$ generated by quadrics. For $d=n=3$, the plane cubic $G$ has $G^{\perp}$ generated by quadrics if and only if it is concise and has no degree 3 minimal generators or, equivalently, $G$ is not a limit of direct sums; see Table 1 . In particular, the general plane cubic has its apolar ideal generated by quadrics. For $d=n \geq 4$, the general form produces a compressed algebra (see the proof of Lemma 3.3) and thus has no quadratic generators in the apolar ideal.

\section{Variation in Families}

If $F_{t} \rightarrow F$, then it does not necessarily follow that $F_{t}^{\perp} \rightarrow F^{\perp}$ or $A_{F_{t}} \rightarrow A_{F}$ as flat families. For example, $x^{d}+t y^{d} \rightarrow x^{d}$ as $t \rightarrow 0$, but $\left(x^{d}\right)^{\perp}=\left\langle\alpha^{d+1}, \beta\right\rangle$ is not the flat limit of the ideals $\left(x^{d}+t y^{d}\right)^{\perp}=\left\langle t \alpha^{d}-\beta^{d}, \alpha \beta\right\rangle$. This can also occur if all polynomials in the family are concise, for example, $x^{d}+y^{d}+t(x+y)^{d} \rightarrow$ $x^{d}+y^{d}$ as $t \rightarrow 0$.

When $\left\{F_{t}\right\}$ is a family of polynomials such that $\left\{F_{t}^{\perp}\right\}$ is a flat family, we say that $\left\{F_{t}\right\}$ is an apolar family and $F_{t} \rightarrow F_{0}$ is an apolar limit. It is equivalent to say that $\left\{A_{F_{t}}\right\}$ is a flat family.

Since we only consider homogeneous polynomials, $\left\{F_{t}\right\}$ is an apolar family if and only if the Hilbert functions of the $A_{F_{t}}$ are locally constant [18, Exer. 20.14]. When this holds, in particular their sum, the length of $A_{F_{t}}$, is locally constant. On the other hand, the values of the Hilbert function are lower semicontinuous in $t$ since they are the ranks of catalecticants, which are linear maps depending regularly on $t$. Thus, if length $\left(A_{F_{t}}\right)$ is constant in $t$, then the Hilbert function must also be constant. We emphasize that this implication is dramatically false if we consider flat families of apolar algebras of nonhomogeneous polynomials; see, for instance, [27] or [11].

REMARK 5.1. Families of homogeneous polynomials $F_{t}$ such that the algebras $A_{F_{t}}$ have fixed Hilbert function are intensively studied. If $T$ is a finite sequence of positive integers, then the set of all homogeneous polynomials of degree $d$ with 
Hilbert function $T$ is denoted in the literature by $\operatorname{Gor}(T)$; see, for instance, [29]. In particular, a family $F_{t}$ is an apolar family if and only if for some $T$ we have $F_{t} \in \operatorname{Gor}(T)$ for all $t$.

Proposition 5.2. Every concise limit of cubic forms is an apolar limit.

Proof. The Hilbert function of the apolar algebra of any concise cubic form in $n$ variables is $1, n, n, 1$, so every concise cubic form has apolar length $2 n+2$, and every family of concise cubic forms is automatically an apolar family.

Proposition 5.2 shows that when $d=3$, ApoLim $\cap$ Con $=$ ApoEqu $\cap$ Con. We will show that for some $n$ and sufficiently large $d$, ApoLim $\cap$ Con $\varsubsetneqq$ ApoEqu $\cap$ Con. Then we will show that for $n=3$, once again ApoLim $\cap \mathrm{Con}=\mathrm{ApoEqu} \cap \mathrm{Con}$. First, we introduce cactus rank and use it to examine some cases in which we can show that a form $F$ has numerous equipotent apolar generators.

\subsection{Cactus Rank and Number of Equipotent Apolar Generators}

In this section, we examine some cases in which we can show that a form $F$ not only has a equipotent apolar generator, but in fact has several such generators. Throughout this section, we assume that $n=\operatorname{dim} V \geq 2$.

Proposition 5.3. Suppose that $F$ is concise and that the Waring rank $r(F)=n$. Then $F$ is an $n$-fold direct sum, and $F$ has at least $n-1$ equipotent apolar generators. Furthermore, if $d>2$, then $F$ has exactly $n-1$ equipotent apolar generators.

Proof. Up to a choice of coordinates, $F$ is the equation of the Fermat hypersurface.

Proposition 5.4. Suppose that $F$ is concise and that the border rank $b r(F)=n$. Then $F$ is a limit of $n$-fold direct sums, and $F$ has at least $n-1$ equipotent apolar generators.

Proof. $F$ is a limit of polynomials as in Proposition 5.3. The second statement follows by Theorem 4.8.

There is another notion of rank of polynomials, namely the cactus rank [43]. It is also called the scheme length in [29, Def. 5.1]. The cactus rank of $F \in S^{d} V$ is the minimal length of a zero-dimensional subscheme $R \subset \mathbb{P V}$ such that $[F] \in$ $\left\langle v_{d}(R)\right\rangle$, or equivalently $I(R) \subset F^{\perp}$ [8, Prop. 3.4(vi)]. We prove an analogue of Propositions 5.3 and 5.4 for cactus rank.

Theorem 5.5. Suppose that $F$ is concise and that the cactus rank $\operatorname{cr}(F)=n$. Then $F$ is a limit of direct sums, and $F$ has at least $n-1$ equipotent apolar generators.

Unlike in Propositions 5.3 and 5.4, we do not claim that $F$ is a limit of $n$-fold direct sums. 
This theorem is proven in three steps. The first step (Lemma 5.6) is the same statement but with an extra assumption that $d \geq n+1$. In the second step, we use the first step to prove a property about syzygies of zero-dimensional schemes embedded in a concisely independent way, which might be of interest on its own. In the final step, we use the syzygies of schemes to prove the theorem.

To obtain a number of minimal generators in some degree, we compare two ideals $J \subset I \subset T$ (e.g., $I=F^{\perp}$ ), where $J$ is generated by $I_{\leq \delta}$. Then we compare the Hilbert functions of $T / I$ and $T / J$. The smallest integer $d$ where $h_{T / I}(d) \neq$ $h_{T / J}(d)$ is a degree in which there must be a minimal generator of $I$; in fact, there are at least $h_{T / J}(d)-h_{T / I}(d)$ minimal generators of degree $d$.

Lemma 5.6. With $F$ as in Theorem 5.5, if in addition $d \geq n+1$, then $h_{A_{F}}$, the Hilbert function of $A_{F}$, is $(1, n, n, \ldots, n, 1,0, \ldots)$, and $F^{\perp}$ has exactly $n-1$ minimal generators in degree $d$.

Proof. Consider an ideal $I \subset T$ defining the scheme realizing the cactus rank of $F$. That is, $I$ is a saturated homogeneous ideal, $I \subset F^{\perp}$, and $B=T / I$ is a graded algebra with constant Hilbert polynomial equal to $\operatorname{cr}(F)=n$. Let $I^{\prime}=I_{\leq n}$ be the ideal generated by the forms in $I$ of degree less than or equal to $n$, and let $B^{\prime}=T / I^{\prime}$.

First, note that we have the following inequality of Hilbert functions: $h_{A_{F}} \leq$ $h_{B} \leq n$. Since $F$ is concise, $n=h_{A_{F}}(1)=h_{A_{F}}(d-1)$. Thus, $h_{B}(1)=n$, and since $h_{B}$ is nondecreasing [38, Rem. 2.8], we must have $h_{B}(i)=n$ for all $i \geq 1$. In particular, $h_{B^{\prime}}(n)=h_{B}(n)=n$ since $I_{n}^{\prime}=I_{n}$, and $h_{B^{\prime}}(n+1) \geq h_{B}(n+1)=n$. On the other hand, by Macaulay's growth theorem [25, Thm. 3.3] or [8, Cor. 5.1] we have $h_{B^{\prime}}(n+1) \leq h_{B^{\prime}}(n)$. Thus, $B^{\prime}$ realizes the maximal possible growth of a Hilbert function from $h_{B^{\prime}}(n)=h_{B^{\prime}}(n+1)$ onwards, and hence by Gotzmann's persistence theorem [25, Thm. 3.8] or [8, Cor. 5.3] we have $h_{B^{\prime}}(i)=n=h_{B}(i)$ for all $i \geq n$. Thus, $I_{i}^{\prime}=I_{i}$ for all $i \geq n$. This shows that the ideal $I$ is generated by $I_{\leq n}$.

By Macaulay's growth theorem we have $n=h_{A_{F}}(d-1) \leq h_{A_{F}}(d-2) \leq$ $\cdots \leq h_{A_{F}}(n) \leq n$. In particular, $I$ and the ideal generated by $I_{n}=\left(F^{\perp}\right)_{n}$ agree in degrees $n, n+1, \ldots, d-1$. However, $h_{A_{F}}(d)=1$, whereas $h_{B}(d)=n$. Thus, $F^{\perp}$ needs exactly $n-1$ minimal generators in degree $d$. Moreover, $I$ is saturated, and $F_{\leq n}^{\perp}$ is a saturation of $\left(F^{\perp}\right)_{n}=I_{n}$; hence,

$$
F_{\leq n}^{\perp}=\left(\left(F^{\perp}\right)_{n}\right)^{\text {sat }}=\left(I_{n}\right)^{\text {sat }} \subset I^{\text {sat }}=I \subset F^{\perp} .
$$

Therefore, $F^{\perp}$ and $I$ agree up to degree $d-1$, and the Hilbert function of $A_{F}$ is $(1, n, n, \ldots, n, 1)$.

We will consider $R \subset \mathbb{P} V$, a zero-dimensional locally Gorenstein subscheme. Such schemes arise naturally when considering cactus rank. Namely, it follows from [8, Lemma 2.3] that if $\operatorname{cr}(F)=n$, then there exists a zero-dimensional locally Gorenstein subscheme $R$ such that length $R=n$ and $F \in\left\langle v_{d}(R)\right\rangle$. Here we will study such $R$ that are embedded into $\mathbb{P} V$ in a concisely independent way, that is, length $R=\operatorname{dim} V$, and $R$ is not contained in any hyperplane. Note that every 
finite scheme can be embedded in a concisely independent way: By, for example, [9, Lemma 2.3], if $R \subset \mathbb{P} V$ is a finite scheme of length $r$, then the Veronese reembedding $v_{r-1}(R) \subset \mathbb{P}\left(S^{r-1} V\right)$ spans an $(r-1)$-dimensional projective subspace in which $R$ is embedded concisely independently.

EXAmPle 5.7. Three points on a line $\mathbb{P}^{1}$ are not concisely independent. Three points on a plane $\mathbb{P}^{2}$ in a general position are embedded in a concisely independent way. Three points in space $\mathbb{P}^{3}$ are not concisely independent.

ExAmPle 5.8. Suppose $R=\operatorname{Spec} \mathbb{C}[\epsilon] /\left\langle\epsilon^{r}\right\rangle$. Then a concisely independent embedding of $R$ in $\mathbb{P}^{r-1}$ is given by the ideal generated by $2 \times 2$ minors of the matrix

$$
M=\left(\begin{array}{ccccc}
\alpha_{1} & \alpha_{2} & \cdots & \alpha_{r-1} & \alpha_{r} \\
\alpha_{2} & \alpha_{3} & \cdots & \alpha_{r} & 0 \\
\vdots & \vdots & & & \\
\alpha_{r-1} & \alpha_{r} & \cdots & 0 & 0 \\
\alpha_{r} & 0 & \cdots & 0 & 0
\end{array}\right)
$$

Here $\alpha_{1}, \ldots, \alpha_{r}$ are homogeneous coordinates on $\mathbb{P}^{r-1}$.

Example 5.9. Suppose $R=\operatorname{Spec} \mathbb{C}[\alpha, \beta] /\left\langle\alpha^{2}, \beta^{2}\right\rangle$. Then a concisely independent embedding of $R$ in $\mathbb{P}^{3}$ is given by the ideal $\left\langle\alpha^{2}, \beta^{2}, \gamma^{2}, \alpha \gamma, \beta \gamma, \alpha \beta-\gamma \delta\right\rangle$. Here $\alpha, \beta, \gamma, \delta$ are homogeneous coordinates on $\mathbb{P}^{3}$.

We remark that a concisely independent embedding of a finite scheme is unique up to linear change of variables: it is the embedding of $R=\operatorname{Spec} A$ into $\mathbb{P}(A)$, where the algebra $A$ is treated as a complex vector space. It is also maximal in the following sense: any other map $R \rightarrow \mathbb{P}^{k}$ can be obtained as a composition $R \hookrightarrow \mathbb{P}(A)-\rightarrow \mathbb{P}^{k}$, where $R \hookrightarrow \mathbb{P}(A)$ is the concisely independent embedding, and $\mathbb{P}(A) \rightarrow \mathbb{P}^{k}$ is a rational linear map (a projection followed by an inclusion).

The following example is tailor made for our purposes, as a preparation to Example 5.18.

EXAMPLE 5.10. Suppose that $G$ is a concise cubic in six variables $x_{1}, \ldots, x_{6}$. Let $R=\operatorname{Spec} A_{G}$ be the zero-dimensional Gorenstein scheme of length 14 determined by $G$. We will now describe in some detail a concisely independent embedding of $R$ into $\mathbb{P}^{13}=\mathbb{P} V$, where $V=\left\langle x_{1}, \ldots, x_{6}, y_{1}, \ldots, y_{6}, z, w\right\rangle$, and $T=\mathbb{C}\left[\alpha_{1}, \ldots, \alpha_{6}, \beta_{1}, \ldots, \beta_{6}, \gamma, \delta\right]$ is the coordinate ring. This embedding will play a role in Example 5.18, our explicit example of a homogeneous form which is a limit of direct sums but not an apolar limit of direct sums.

Consider the ideal $I$ generated by all of the following:

(i) The apolar ideal of $G$ in $\mathbb{C}\left[\alpha_{1}, \ldots, \alpha_{6}\right]$. This provides 15 quadric minimal generators and perhaps some cubics.

(ii) 30 quadrics $\alpha_{i} \beta_{j}$ for $i \neq j, i, j \in\{1, \ldots, 6\}$.

(iii) 13 quadrics $\alpha_{i} \gamma, \beta_{i} \gamma, \gamma^{2}$ for $i \in\{1, \ldots, 6\}$.

(iv) 21 quadrics $\beta_{i} \beta_{j}$ for $i, j \in\{1, \ldots, 6\}$. 
(v) 6 quadrics $\alpha_{i} \beta_{i}-\gamma \delta$ for $i \in\{1, \ldots, 6\}$.

(vi) 6 quadrics obtained in the following way: For $i \in\{1, \ldots, 6\}$, let $\Theta_{i} \in$ $\mathbb{C}\left[\alpha_{1}, \ldots, \alpha_{6}\right]$ be a quadric such that $\left.\Theta_{i}\right\lrcorner G=x_{i}$ (these quadrics exist since $G$ is concise with respect to $\left.\left\langle x_{1}, \ldots, x_{6}\right\rangle\right)$. Then include in $I$ the quadrics $\Theta_{i}-\beta_{i} \delta$.

Altogether, we obtain 91 quadrics and perhaps some cubics (depending on $G$ ). The radical of the homogeneous ideal $I$ is generated by

$$
\sqrt{I}=\left\langle\alpha_{1}, \ldots, \alpha_{6}, \beta_{1}, \ldots, \beta_{6}, \gamma\right\rangle
$$

since $\alpha_{i}^{4} \in I$ by (i), $\beta_{i}^{2} \in I$ by (iv), and $\gamma^{2} \in I$ by (iii). Thus, the projective scheme defined by $I$ is supported at the single point $[w] \in \mathbb{P} V$, which is contained in the open subset $\delta \neq 0$. Evaluating the generators of $I$ at $\delta=1$, the reader can easily check that the scheme supported at $[w]$ is isomorphic to $R$ and also that there are no linear forms in $T_{1}$ that contain this scheme.

Also, it is not difficult to see that the Hilbert function of $T / I$ is $(1,14,14,14$, ...). We combine this information with the fact that the Hilbert function of a saturated ideal is nondecreasing; see [38, Rem. 2.8]. We conclude that $I$ is the saturated ideal defining $R \subset \mathbb{P} V$, and the embedding of $R$ is concisely independent because $I_{1}=0$ and length $R=\operatorname{dim} V=14$.

Finally, we remark that for general $G \in S^{3} \mathbb{C}^{6}$, the scheme $R$ is a shortest nonsmoothable Gorenstein scheme. See [29, Lemma 6.21], where it is shown that $R$ is nonsmoothable, and $[14 ; 11]$, where it is shown that all shorter Gorenstein schemes are smoothable.

Proposition 5.11. Suppose that $R$ is a finite locally Gorenstein scheme, length $R=n$, and $R \subset \mathbb{P} V$ is concisely independent. Let $J \subset T$ be the saturated homogeneous ideal of $R$. Then $\beta_{n-1, n}(J) \geq n-1$.

Proof. Consider a general $F \in\left\langle v_{d}(R)\right\rangle$ for some $d \geq 2 n$. It follows from [8, Lemma 2.3] that $F$ is not contained in $\left\langle v_{d}(Q)\right\rangle$ for any $Q \varsubsetneqq R$. It further follows from [9, Cor. 2.7] that $R$ is determined by $F$. Namely, $R$ is the unique subscheme of $\mathbb{P} V$ of length $n$ such that $F \in\left\langle v_{d}(R)\right\rangle$. Also, $\operatorname{cr}(F)=n$. By [8, Thm. 1.6], $J=\left(F^{\perp}\right)_{\leq n}$. In particular, $h_{A_{F}}(1)=h_{T / J}(1)=n$, that is, $F$ is concise.

By Lemma 5.6 and Gorenstein symmetry (2) we have $\beta_{n-1, n}\left(F^{\perp}\right) \geq n-1$. The syzygies involve only quadratic generators of $F^{\perp}$, so they also exist in $\left(F^{\perp}\right)_{\leq n}=J$, and $\beta_{n-1, n}(J) \geq n-1$, as claimed.

Proof of Theorem 5.5. Let $R \subset \mathbb{P} V$ be a locally Gorenstein scheme of length $n$ such that $F \in\left\langle v_{d}(R)\right\rangle$, whose existence is guaranteed by the definition of cactus rank and [8, Lemma 2.3]. Let $J \subset T$ be the homogeneous saturated ideal defining $R$. We have $J \subset F^{\perp}$, and thus

$$
\beta_{n-1, n}\left(F^{\perp}\right) \geq \beta_{n-1, n}(J) \geq n-1
$$

by Lemma 2.9 and Proposition 5.11. By Gorenstein symmetry (2) the ideal $F^{\perp}$ must have at least $n-1$ minimal generators of degree $d$, as claimed. 


\subsection{Cleavable and Uncleavable Schemes}

In this section, we give examples of limits of direct sums that cannot be obtained as apolar limits of direct sums; these are points in ApoEqu that are not in ApoLim.

For a field $\mathbb{k}$, let $n_{0, \mathbb{k}}$ be the minimal length of a nonsmoothable locally Gorenstein scheme defined over $\mathbb{k}$. Recall that we are working over the field $\mathbb{C}$, in particular, in characteristic 0 . As we will see, for our purposes, we do not need to know the value of $n_{0, \mathbb{k}}$, just that there is such a value. For simplicity, we write $n_{0}$

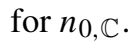

Although the value of $n_{0}$ does not matter for us, it turns out that in characteristic 0 this value has been determined very recently. It is well known that $12 \leq n_{0} \leq 14$ (see [8, Sect. 6, Sect. 8.1] for an overview and references and the recent work [14] proving $n_{0} \neq 11$ ). Even more recently, Casnati, Jelisiejew, and Notari [11, Thms. A and B] have shown that $n_{0}=14$. That is, they prove that all Gorenstein schemes of length at most 13 are smoothable. This had been predicted by A. Iarrobino (private communication). See also [31] for a related partial result.

For the remainder of this section, we work in characteristic 0 , so the reader may take $n_{0}$ to be 14 .

Proposition 5.12. Let $n=\operatorname{dim} V \geq n_{0}$, and $d \geq 2 n-1$. Then there exist concise polynomials $F \in S^{d} V$ with $\operatorname{cr}(F)=n$ but $\operatorname{br}(F)>n$.

Proof. Let $R$ be any nonsmoothable Gorenstein scheme of length $n$. Embed $R \subset$ $\mathbb{P} V$ in a concisely independent way. Let $F \in\left\langle v_{d}(R)\right\rangle$ be a general element. Then $F$ is not contained in $\left\langle v_{d}\left(R^{\prime}\right)\right\rangle$ for any $R^{\prime} \varsubsetneqq R$ by [9, Lemma 3.5(iii)]. By [9, Cor. 2.7] we cannot have $F \in\left\langle v_{d}(Q)\right\rangle$ for any scheme $Q \subset \mathbb{P} V$ of length less than $n$, so $\operatorname{cr}(F)=n$. For the same reason, since $R$ is not smoothable, there exists no smoothable scheme $Q \subset \mathbb{P} V$ of length at most $n$ with $F \in\left\langle v_{d}(Q)\right\rangle$. Were $\operatorname{br}(F) \leq n$, then there would have to be such a $Q$; see, for example, [3, Prop. 11], [9, Lem. 2.6], or [8, Prop. 2.5]. Thus, $\operatorname{br}(F)>n$.

REMARK 5.13. We have seen that if $F$ has an equipotent apolar generator, then $F$ is a limit of direct sums. Also, we have seen that if $F$ is a concise form in $n$ variables and $F$ is a limit of direct sums of $n$ terms, then $F$ has $n-1$ equipotent apolar generators. Now, a form $F$ as in Proposition 5.12 has $n-1$ equipotent apolar generators by Theorem 5.5, so it is a limit of direct sums, but it is not necessarily a limit of direct sums of $n$ terms.

Thus, the closure of the locus of Fermat polynomials is contained in the locus of forms with $n-1$ equipotent apolar generators, but this containment can be strict.

Proposition 5.14. Let $n<n_{0}$. Then every homogeneous concise polynomial $F \in$ $S^{d} V$ with $\operatorname{cr}(F)=n$ has $\operatorname{br}(F)=n$.

Proof. By [8, Thm. 1.4(i)] we have $\operatorname{br}(F) \leq n$. Since $F$ is concise, we also have $\operatorname{br}(F) \geq n$.

We introduce some terminology about zero-dimensional schemes. 
Definition 5.15. Suppose that $\mathcal{R} \rightarrow B$ is a flat family of zero-dimensional schemes, $b \in B$ is a closed point, and $R=\mathcal{R}_{b}$ is the special fiber over $b$. We say that $\mathcal{R} \rightarrow B$ is a cleaving of $R$ if the base $B$ is irreducible, the special fiber $R$ is supported at a single point, and the general fiber is not supported at a single point. If $R$ admits a cleaving, then we say that $R$ is cleavable. Otherwise, that is, if $R$ is a finite scheme supported at a single point that does not admit any cleaving, then we say that $R$ is uncleavable.

We remark that in [11] cleavable schemes are called limit-reducible, and uncleavable schemes are called strongly nonsmoothable. In [28], a component of the Hilbert scheme containing uncleavable schemes is called an elementary component. Note however that not every scheme that belongs to an elementary component is uncleavable since this component intersects also other components of the Hilbert scheme.

Lemma 5.16. The following are elementary properties of cleavings and (un-)cleavable schemes.

(i) A single reduced point is uncleavable. All other smoothable schemes are cleavable.

(ii) A general fiber of any cleaving of a zero-dimensional Gorenstein scheme supported at a single point is a Gorenstein scheme. (More generally, every deformation of a finite Gorenstein scheme is Gorenstein.)

(iii) Every nonsmoothable Gorenstein scheme of length $n_{0}=14$ is local (that is, supported at a single point) and uncleavable.

(iv) Every Gorenstein scheme of length less than $n_{0}$ is cleavable, unless it is a single reduced point.

Proof. The first property is clear. To be Gorenstein is an open condition on the Hilbert scheme, thus the second property follows.

Also the third property is straightforward -if there exists a cleaving of a Gorenstein scheme of length $n_{0}$, then $R$ is a flat limit of a disjoint union of two shorter Gorenstein schemes. By the definition of $n_{0}$ both shorter schemes must be smoothable. Thus, $R$ is smoothable.

The final property is clear since all smoothable schemes are flat limits of disjoint points, and hence they admit a cleaving.

Note that not every nonsmoothable Gorenstein scheme is uncleavable. Potentially, it could happen that every nonsmoothable Gorenstein scheme of some fixed length admits a cleaving to a disjoint union of two shorter schemes, at least one of which is nonsmoothable. Thus, whereas every $n \geq n_{0}$ is clearly the length of a nonsmoothable Gorenstein scheme, it could potentially happen that some $n>n_{0}$ is not the length of any uncleavable Gorenstein scheme.

Let us briefly remark that there exist uncleavable schemes of any (sufficiently high) finite length if we drop the assumption of Gorenstein [46, Thm. 2]. For the Gorenstein case, see [29, Lem. 6.21], in which it is shown that for $r \in$ $\{6,8,9,10,11,12\}$, general Gorenstein schemes with Hilbert function $(1, r, r, 1)$ 
are uncleavable (these have lengths $n \in\{14,18,20,22,24,26\}$ ). It is expected that the statement holds for every $r \geq 8$. (Gorenstein schemes with Hilbert function $(1,7,7,1)$ are smoothable [4].)

But it is beyond the scope of this paper to determine all possible lengths of uncleavable Gorenstein schemes. For the purpose of Theorem 1.8, it is enough that there exists such a length, namely $n_{0}=14$.

So, let $R$ be an uncleavable Gorenstein scheme of length $n_{1}>1$. In particular, by Lemma 5.16 we have $n_{1} \geq n_{0}$. For example, we may choose $R$ such that $n_{1}=n_{0}$. In characteristic 0 , another possible value of $n_{1}>14$ would be the minimal length of a nonsmoothable Gorenstein scheme contained in $\mathbb{P}^{5}$ (or, respectively, in $\mathbb{P}^{4}$ ). It is known that such $n_{1} \leq 42$ (respectively, $n_{1} \leq 140$ ).

Proposition 5.17. Suppose $n=n_{1}$ and $d \geq 2 n_{1}$, with $R \subset \mathbb{P} V$ a concisely independently embedded uncleavable Gorenstein scheme of length $n_{1}$. Let $F \in S^{d} V$ be a concise polynomial such that $\operatorname{cr}(F)=n_{1}$ and $F \in\left\langle v_{d}(R)\right\rangle$. Then $F$ is a limit of direct sums, but it is not an apolar limit of direct sums.

For example, if $n_{1}=n_{0}$, then take $F$ with $\operatorname{cr}(F)=n_{0}$ and $\operatorname{br}(F)>n_{0}$. See Example 5.18 for an explicit example of such a polynomial $F$.

Proof of Proposition 5.17. Suppose on the contrary that $F$ is an apolar limit of direct sums $F_{t}=G_{t}+H_{t}$. The Hilbert function of $A_{F}$ is $(1, n, n, \ldots, n, 1,0, \ldots)$ by Lemma 5.6. Consider the two Hilbert functions $h_{A_{G_{t}}}$ and $h_{A_{H_{t}}}$. We must have $h_{A_{G_{t}}}(k)+h_{A_{H_{t}}}(k)=n$ for all $1 \leq k \leq d-1$. In particular, since $h_{A_{G_{t}}}(k)$, $h_{A_{H_{t}}}(k) \geq 1$, we have $h_{A_{G_{t}}}(k), h_{A_{H_{t}}}(k) \leq n-1$ for all $1 \leq k \leq d-1$. By [8, Lem. 5.2] we must have $h_{A_{G_{t}}}(k)=(1, a, a, \ldots, a, 1,0, \ldots)$ and $h_{A_{H_{t}}}(k)=$ $(1, b, b, \ldots, b, 1,0, \ldots)$ for all $t$ close to 0 , where $a+b=n$.

By [8, Thm. 1.6], $G_{t} \in\left\langle v_{d}\left(Q_{t}^{\prime}\right)\right\rangle$ and $H_{t} \in\left\langle v_{d}\left(Q_{t}^{\prime \prime}\right)\right\rangle$ for schemes $Q_{t}^{\prime}$ and $Q_{t}^{\prime \prime}$ of length $a$ and $b$, respectively. Denote the flat limit $Q=\lim _{t \rightarrow 0}\left(Q_{t}^{\prime} \sqcup Q_{t}^{\prime \prime}\right)$. The length of $Q$ is $a+b=n$. Moreover, for each $t$ close but not equal to zero, $Q_{t}^{\prime}$ and $Q_{t}^{\prime \prime}$ are embedded (respectively) into disjoint linear subspaces $\mathbb{P}_{t}^{a-1}$ and $\mathbb{P}_{t}^{b-1}$. Thus, the defining ideal $I\left(Q_{t}^{\prime} \sqcup Q_{t}^{\prime \prime}\right)$ of $Q_{t}^{\prime} \sqcup Q_{t}^{\prime \prime}$ satisfies

$$
I\left(Q_{t}^{\prime} \sqcup Q_{t}^{\prime \prime}\right)=I\left(Q_{t}^{\prime}\right) \cap I\left(Q_{t}^{\prime \prime}\right)=\left(G_{t}^{\perp}\right)_{\leq n} \cap\left(H_{t}^{\perp}\right)_{\leq n} .
$$

The last equality $I\left(Q_{t}^{\prime}\right)=\left(G_{t}^{\perp}\right)_{\leq n}$ (and analogously for $Q_{t}^{\prime \prime}$ and $H_{t}$ ) follows from [8, Thm. 1.6(iii)]. Since $F_{t}=G_{t}+H_{t}$ is a direct sum, by Lemma 1.12 we have $\left(G_{t}^{\perp}\right)_{\leq n} \cap\left(H_{t}^{\perp}\right)_{\leq n}=\left(F_{t}^{\perp}\right)_{\leq n}$. Since we are considering an apolar limit, we may pass to the limit with ideals:

$$
J=\lim _{t \rightarrow 0}\left(I\left(Q_{t}^{\prime} \sqcup Q_{t}^{\prime \prime}\right)\right)=\lim _{t \rightarrow 0}\left(F_{t}^{\perp}\right)_{\leq n}=\left(F^{\perp}\right)_{\leq n} .
$$

The ideal $J$ is a homogeneous ideal defining $Q=\lim _{t \rightarrow 0}\left(Q_{t}^{\prime} \sqcup Q_{t}^{\prime \prime}\right)$, though at this point potentially $J$ is not saturated. However, using $J=\left(F^{\perp}\right)_{\leq n}$ and by [8, Thm. 1.6(iii)] applied to $F$ we have that the ideal $J$ must be saturated and $F \in\left\langle v_{d}(Q)\right\rangle$. By the uniqueness in [8, Thm. 1.6(ii)] we have $R=Q$, which is a contradiction since $Q$ is a limit of smaller disjoint schemes, that is, $Q$ is cleavable, whereas $R$ is not. 
In the case where $n_{1}=n_{0}$ and $Q$ is smoothable of length $n_{0}$, the previous considerations imply that the border rank of $F$ is (at most) $n$, a contradiction with our assumption $\operatorname{br}(F)>n$.

EXAmPLE 5.18. Let $d \geq 3$, let $G$ be a general homogeneous cubic in six variables $x_{1}, \ldots, x_{6}$, and let

$$
\begin{aligned}
F= & (d-2) z^{d-3} G+z^{d-2}\left(x_{1} y_{1}+x_{2} y_{2}+x_{3} y_{3}+x_{4} y_{4}+x_{5} y_{5}+x_{6} y_{6}\right) \\
& +\frac{1}{d-1} z^{d-1} w .
\end{aligned}
$$

Consider the concisely independent scheme $R$ defined from $G$ as in Example 5.10. Then $F$ is apolar to $R$, which can be verified by acting on $F$ with the generators (i)-(vi) of Example 5.10. Thus, $F \in\left\langle v_{d}(R)\right\rangle$. Moreover, since $G$ is general, in characteristic $0, R$ is a shortest nonsmoothable Gorenstein scheme; see [11, Thm. A] and [29, Lem. 6.21]. In particular, $R$ is uncleavable by Lemma 5.16. Thus, by Proposition 5.17, $F$ is a limit of direct sums but not an apolar limit of direct sums.

\subsection{Apolar Limits in the Plane}

We show that in the plane, ApoLim $=$ ApoEqu. Recall that when considering forms in three variables, we write $S=\mathbb{C}[x, y, z]$ and $T=\mathbb{C}[\alpha, \beta, \gamma]$.

TheOREm 5.19. Let $F$ be a concise form of degree $d$ in $n=3$ variables having an equipotent apolar generator. Then $F$ is an apolar limit of direct sums.

Note that, in contrast to the situation of Proposition 5.2, where every family of direct sum cubic forms having as the limit a concise cubic form must be an apolar family, we do not claim here that every family $F_{t} \rightarrow F$ is necessarily an apolar family. Rather, we claim only that there exists some apolar family of direct sums $F_{t} \rightarrow F$.

As we will see in the proof of Theorem 5.19, "typically" (here we do not want to specify precisely what does "typically" mean; see the proof below for an explicit statement), the limit indicated in Example 4.9 provides an apolar limit. However, this limit does not work in all cases, as illustrated by the following example.

EXAmPLE 5.20. Consider the following sextic in three variables:

$$
F=x y^{5}+y^{3} z^{3} \text {. }
$$

As indicated by Example 4.9, this is a limit of direct sums. Indeed,

$$
F_{t}=\frac{1}{t}\left(\left(y+\frac{1}{6} t x\right)^{6}+t y^{3} z^{3}-y^{6}\right)
$$

is a family of homogeneous polynomials ( $t$ is a parameter of the family) with $F_{0}=F$, and $F_{t}$ for $t \neq 0$ after an easy coordinate change becomes

$$
x^{6}+y^{3} z^{3}-y^{6} \text {. }
$$


In particular, $F_{t}$ for $t \neq 0$ is a direct sum and the Hilbert function of $A_{F_{t}}$ is 1,3 , 4, 5, 4, 3, 1, whereas the Hilbert function of $A_{F}=A_{F_{0}}$ is $1,3,4,4,4,3,1$. Thus, the family $F_{t}$ is not an apolar family. However, another family

$$
\frac{1}{t}\left(\left(y+\frac{1}{6} t x\right)^{6}-y^{6}+t y^{3} z^{3}-\frac{1}{400} t^{2} z^{6}\right)
$$

presents $F$ as an apolar limit of direct sums.

Proof of Theorem 5.19. By Example 4.9, either $F$ is a direct sum, in which case the statement is trivial, or else after a change of coordinates, $F=x y^{d-1}+G(y, z)$. Write $G(y, z)=\sum_{q=0}^{d}\left(\begin{array}{l}d \\ q\end{array}\right) a_{q} y^{q} z^{d-q}$.

Recall that $T=\mathbb{C}[\alpha, \beta, \gamma]$ is the dual ring to $S=\mathbb{C}[x, y, z]$. First, we find the Hilbert function of $A_{F}$. In order to reduce subscripts, we write $h_{F}$ for the Hilbert function of $A_{F}$. We have $h_{F}(0)=h_{F}(d)=1$. We claim that for $1 \leq k \leq d-1$, $h_{F}(k)=h_{\left.\gamma^{2}\right\lrcorner G}(k-1)+2$.

Recall that for a form $H \in S_{e}$ of degree $e$ and an integer $\left.i \geq 0, T_{i}\right\lrcorner H$ is the linear subspace $\left.\left.T_{i}\right\lrcorner H=\{\Theta\lrcorner H \mid \Theta \in T_{i}\right\} \subseteq S_{e-i}$.

First, $\left.h_{\left.\gamma^{2}\right\lrcorner G}(k-1)=\operatorname{dim}\left(T_{d-k-1} \gamma^{2}\right\lrcorner G\right)$, and $\left.T_{d-k-1} \gamma^{2}\right\lrcorner G$ is spanned by, for $0 \leq i \leq d-k-1$,

$$
\begin{aligned}
\left.\beta^{i} \gamma^{d-k-1-i} \gamma^{2}\right\lrcorner G & =\frac{d !}{(k-1) !} \sum_{q=i}^{i+k-1}\left(\begin{array}{c}
k-1 \\
q-i
\end{array}\right) a_{q} y^{q-i} z^{k+i-q-1} \\
& =\frac{d !}{(k-1) !} \sum_{j=0}^{k-1}\left(\begin{array}{c}
k-1 \\
j
\end{array}\right) a_{j+i} y^{j} z^{k-j-1}
\end{aligned}
$$

So $h_{\left.\gamma^{2}\right\lrcorner G}(k-1)=\operatorname{rank} M$ where $M_{i j}=\left(\begin{array}{c}k-1 \\ j\end{array}\right) a_{j+i}$ for $0 \leq i \leq d-k-1,0 \leq$ $j \leq k-1$.

Meanwhile, $\left.h_{F}(k)=\operatorname{dim} T_{d-k}\right\lrcorner F$, and $\left.T_{d-k}\right\lrcorner F$ is spanned by $\left.T_{d-k-1} \gamma\right\lrcorner F=$ $\left.T_{d-k-1} \gamma\right\lrcorner G$ together with $\left.\left\{\alpha^{j} \beta^{d-k-j}\right\lrcorner F \mid 0 \leq j \leq d-k\right\}$. Note that $\left.\alpha^{2}\right\lrcorner F=0$. We have

$$
\left.\left.\left.\alpha \beta^{d-k-1}\right\lrcorner F=\frac{(d-1) !}{k !} y^{k}, \quad \beta^{d-k}\right\lrcorner F=\frac{(d-1) !}{(k-1) !} x y^{k-1}+\beta^{d-k}\right\lrcorner G .
$$

Here, $\left.\beta^{d-k}\right\lrcorner F$ is linearly independent of the other spanning elements since it is the only one with a monomial involving $x$. Also, $\left.T_{d-k-1 \gamma}\right\lrcorner G$ is spanned by, for $0 \leq i \leq d-k-1$,

$$
\begin{aligned}
\beta^{i} \gamma^{d-k-1-i} \gamma-G & =\frac{d !}{k !} \sum_{q=i}^{i+k}\left(\begin{array}{c}
k \\
q-i
\end{array}\right) a_{q} y^{q-i} z^{k+i-q} \\
& =\frac{d !}{k !} \sum_{j=0}^{k}\left(\begin{array}{l}
k \\
j
\end{array}\right) a_{j+i} y^{j} z^{k-j}
\end{aligned}
$$


Thus, $\left.T_{d-k-1} \gamma\right\lrcorner G+\mathbb{C} y^{k}$ is spanned by $y^{k}$ together with, for $0 \leq i \leq d-k-1$,

$$
\sum_{j=0}^{k-1}\left(\begin{array}{l}
k \\
j
\end{array}\right) a_{j+i} y^{j} z^{k-j}
$$

So $\left.\operatorname{dim}\left(T_{d-k-1} \gamma\right\lrcorner G+\mathbb{C} y^{k}\right)=1+\operatorname{rank} N$ where $N_{i j}=\left(\begin{array}{l}k \\ j\end{array}\right) a_{j+i}$ for $0 \leq i \leq$ $d-k-1,0 \leq j \leq k-1$. Note that $N$ is obtained from $M$ by rescaling columns, so rank $M=\operatorname{rank} N$. This proves that $h_{F}(k)=h_{\left.\gamma^{2}\right\lrcorner G}(k-1)+2$, as claimed.

For any $1 \leq r \leq \frac{d}{2}$, let $h^{r}$ be the following function:

$$
h^{r}(k)= \begin{cases}1, & k=0, \\ k+2, & 1 \leq k \leq r-1, \\ r+2, & r \leq k \leq d-r, \\ (d-k)+2, & d-r+1 \leq k \leq d-1, \\ 1, & k=d, \\ 0, & k<0 \text { or } k>d .\end{cases}
$$

We have shown that $h_{F}=h^{r}$ where $\left.r=\operatorname{br}\left(\gamma^{2}\right\lrcorner G\right)$. Let

$$
\left.H^{r}=\left\{F=x y^{d-1}+G(y, z) \mid \operatorname{br}\left(\gamma^{2}\right\lrcorner G\right)=r\right\} .
$$

We claim that $H^{r}$ is irreducible. Write $\hat{\sigma}_{r}\left(v_{d}\left(\mathbb{P}^{1}\right)\right)$ for the affine cone over the $r$ th secant variety. The map taking $F$ to $\left.\gamma^{2}\right\lrcorner G$ is simply the pairing with $\gamma^{2}$ since $\left.\left.\gamma^{2}\right\lrcorner F=\gamma^{2}\right\lrcorner G$ for $F \in H^{r}$. For convenience, we denote this map $\varphi$, so that $\left.\left.\varphi(F)=\gamma^{2}\right\lrcorner F=\gamma^{2}\right\lrcorner G$ for $F \in H^{r}$. Then $\varphi$ maps $H^{r}$ onto $\hat{\sigma}_{r}\left(v_{d-2}\left(\mathbb{P}^{1}\right)\right) \backslash$ $\hat{\sigma}_{r-1}\left(v_{d-2}\left(\mathbb{P}^{1}\right)\right)$, the set of binary forms of border rank $r$, an irreducible variety. Also the fibers of $\varphi$ are irreducible, specifically, copies of $\mathbb{A}^{2}$, since the fiber through $F$ is $\varphi^{-1}(\varphi(F))=\left\{F+a y^{d}+b y^{d-1} z\right\}$. Thus, $H^{r}$ is irreducible.

The claim of the theorem is that for all $F \in H^{r}$, we can obtain $F$ as a limit of direct sums that have the Hilbert function $h^{r}$. In the following paragraph, we are going to prove the claim under the additional assumption that $F$ is a general element of $H^{r}$. Then, in the final paragraph, we are going to use this "generic" case and the irreducibility of $H^{r}$ to conclude the statement for all polynomials $F$ in question.

So suppose that $F \in H^{r}$ is general. Then $\left.\gamma^{2}\right\lrcorner G \in \hat{\sigma}_{r}\left(v_{d-2}\left(\mathbb{P}^{1}\right)\right)$ is also a general element, so $\left.\gamma^{2}\right\lrcorner G=\ell_{1}^{d-2}+\cdots+\ell_{r}^{d-2}$ where the $\left[\ell_{i}\right] \in \mathbb{P}^{1}$ are in general position. In this case, it is easy to integrate $\left.\gamma^{2}\right\lrcorner G$ (twice), and hence $G=c_{1} \ell_{1}^{d}+$ $\cdots+c_{r} \ell_{r}^{d}+a y^{d-1} z+b y^{d}$. Therefore, $F=(x+a z+b y) y^{d-1}+\left(c_{1} \ell_{1}^{d}+\cdots+\right.$ $\left.c_{r} \ell_{r}^{d}\right)$. Let $x^{\prime}=x+a z+b y$. For $t \neq 0$, let $F_{t}^{\prime}=\frac{1}{t}\left(y+\frac{t}{d} x^{\prime}\right)^{d}+\left(c_{1} \ell_{1}^{d}+\cdots+\right.$ $\left.c_{r} \ell_{r}^{d}-\frac{1}{t} y^{d}\right)$. Since the $\ell_{i}$ are general and $r+1 \leq \frac{d+2}{2}$, we have for general $t \neq 0$,

$$
r\left(c_{1} \ell_{1}^{d}+\cdots+c_{r} \ell_{r}^{d}-\frac{1}{t} y^{d}\right)=b r\left(c_{1} \ell_{1}^{d}+\cdots+c_{r} \ell_{r}^{d}-\frac{1}{t} y^{d}\right)=r+1 .
$$

Since $F_{t}^{\prime}$ is a direct sum, $h_{F_{t}^{\prime}}=h^{r}=h_{F}$ (the first equality follows from Proposition 2.10). Thus, $F_{t}^{\prime} \rightarrow F$ is an apolar limit. 
By the argument in the previous paragraph, the irreducible set $H^{r}$ is contained in the Zariski closure of the locus of direct sums with Hilbert function $h^{r}$. So every $F \in H^{r}$ is a limit of direct sums with Hilbert function $h^{r}$, that is, it is an apolar limit of direct sums. This proves the claim of the theorem.

Note that the apolar ideal of a form in three variables is a height 3 Gorenstein ideal and is therefore generated by the principal Pfaffians of a skew-symmetric matrix [7]. Nevertheless, we have not used this information; instead, the key was information about apolar ideals of forms in one less variable. It would be interesting to investigate whether the structure described in [7] can lead to a generalization of Theorem 5.19 for forms in $n=4$ variables; compare with [21].

It would also be interesting to study limits of direct sums of type $(1, n-1)$, $s$-fold direct sums of type $(1,1, \ldots, 1, n-s+1)$, direct sums of type $(1, \ldots, 1$, $2, \ldots, 2)$, and so on. Note however that for limits of direct sums of type $(1, n-1)$, we cannot expect a similar result to Theorem 5.19. This is because for $n=14$, the polynomial $F$ presented in Example 5.18 is a limit of direct sums of type $(1,13)$ but is not an apolar limit, as shown in Example 5.18.

\section{Generalizations and Further Questions}

\subsection{Linear Series}

There are natural generalizations of some of our results to linear series. Let $W \subseteq S^{d} V$ be a linear series. A simultaneous power sum decomposition of $W$ is a collection of linear forms $\ell_{1}, \ldots, \ell_{r}$ such that $W$ is contained in the span of $\ell_{1}^{d}, \ldots, \ell_{r}^{d}$; equivalently, for each $F \in W$, there are scalars $c_{1}, \ldots, c_{r}$ such that $F=\sum c_{i} \ell_{i}^{d}$. The simultaneous Waring rank of $W$, denoted $r(W)$, is the least length $r$ of a simultaneous power sum decomposition.

Let $S=\mathbb{C}[V]=\mathbb{C}\left[x_{1}, \ldots, x_{n}\right]$, where $x_{1}, \ldots, x_{n}$ is a basis for $V$, and let $T=\mathbb{C}\left[V^{*}\right]=\mathbb{C}\left[\alpha_{1}, \ldots, \alpha_{n}\right]$ act on $S$ by letting $\alpha_{i}$ act as the partial differentiation operator $\partial / \partial x_{i}$. The apolar annihilating ideal $W^{\perp} \subset T$ is $W^{\perp}=\{\Theta \mid$ $\Theta\lrcorner F=0, \forall F \in W\}$. That is, $W^{\perp}=\bigcap_{F \in W} F^{\perp}$. The apolar algebra $A_{W}=$ $T / W^{\perp}$ is a level Artinian algebra with socle degree $d$ and type equal to the dimension of $W$, meaning that its socle is entirely in degree $d$ and has dimension equal to $\operatorname{dim} W$. In particular, $A_{W}$ is Gorenstein if and only if $W$ is one-dimensional, that is, spanned by a single form.

There is an apolarity lemma just as in the case of a single form. This is well known to experts; see, for example, [15, Thm. 2.3]. For the reader's convenience, we state it here.

LEMMA 6.1. With notation as before, $\ell_{1}, \ldots, \ell_{r}$ is a simultaneous power sum decomposition of $W$ if and only if the ideal $I=I\left(\left\{\left[\ell_{1}\right], \ldots,\left[\ell_{r}\right]\right\}\right)$ satisfies $I \subseteq W^{\perp}$.

Proof. The decomposition $\ell_{1}, \ldots, \ell_{r}$ is a simultaneous power sum decomposition of $W$ if and only if $W$ lies in the span of the $\ell_{i}^{d}$, if and only if every $F \in W$ can be 
written as a linear combination of the $\ell_{i}^{d}$, if and only if $I \subseteq F^{\perp}$ for every $F \in W$ (by the usual apolarity lemma), and if and only if $I \subseteq \bigcap F^{\perp}=W^{\perp}$.

Thus, $r(W) \geq \operatorname{dim}\left(A_{W}\right)_{a}$ for every $0 \leq a \leq d$ : indeed, if $\ell_{1}, \ldots, \ell_{r}$ is a simultaneous power sum decomposition of $W$ with defining ideal $I$, then $r \geq \operatorname{codim} I_{a} \geq$ $\operatorname{codim}\left(W^{\perp}\right)_{a}=\operatorname{dim}\left(A_{W}\right)_{a}$ for $a>0$ (and it is trivial for $a=0$ ).

There is a generalization of the Ranestad-Schreyer lower bound [43] for Waring rank for linear series, with essentially the same proof as for the case of a single form. We briefly review the proof for completeness.

Proposition 6.2. With notation as before, let length $\left(A_{W}\right)$ be the length of $A_{W}$ and suppose that $W^{\perp}$ is generated in degrees less than or equal to $\delta$. Then $r(W) \geq$ length $\left(A_{W}\right) / \delta$.

Proof. Suppose that $\ell_{1}, \ldots, \ell_{r}$ is a simultaneous power sum decomposition of $W$ with defining ideal $I$. The vanishing locus $V\left(\left(W^{\perp}\right)_{\delta}\right)$ in affine space is just the origin (i.e., a scheme supported at the origin). By Bertini's theorem, the linear series $\left(W^{\perp}\right)_{\delta}$ has no basepoints in projective space. Let $G \in\left(W^{\perp}\right)_{\delta}$ be a general form. Then $G$ does not vanish at any projective point $\left[\ell_{i}\right]$, so the affine hypersurface $V(G)$ does not contain any line that is an irreducible component of $V(I)$. Therefore, by Bezout's theorem the intersection of $V(G)$ and $V(I)$ has degree equal to $\delta r$. But this intersection contains the scheme $V\left(W^{\perp}\right)$, which has length equal to length $\left(A_{W}\right)$. So $\delta r \geq \operatorname{length}\left(A_{W}\right)$.

As in [47, Rem. 3.5], this holds in arbitrary characteristic.

A direct sum decomposition of $W$ is an expression $V=V_{1} \oplus V_{2}$ and subspaces $W_{1} \subseteq S^{d} V_{1}, W_{2} \subseteq S^{d} V_{2}$ such that $W \subset W_{1} \oplus W_{2}$ and the projections $W \rightarrow W_{1}$, $W \rightarrow W_{2}$ are isomorphisms. Equivalently, for a basis $F^{1}, \ldots, F^{k}$ of $W$, each $F^{i}=F_{1}^{i}+F_{2}^{i}$ with $F_{1}^{i} \in W_{1}, F_{2}^{i} \in W_{2}$, and the $F_{1}^{i}$ are linearly independent, and so are the $F_{2}^{i}$.

Now we can generalize some of our results to the case of linear series. Here is a generalization of Theorem 1.1.

Proposition 6.3. If a linear series $W$ of degree $d$ forms admits a direct sum decomposition, then $W^{\perp}$ has at least $s=\operatorname{dim} W$ minimal generators of degree $d$.

Proof. Let $W \subset W_{x} \oplus W_{y}$ be a direct sum decomposition where

$$
\begin{aligned}
W_{x} & \subseteq\left(S^{x}\right)_{d}=\mathbb{C}\left[x_{1}, \ldots, x_{i}\right]_{d}, \quad W_{y} \subseteq\left(S^{y}\right)_{d}=\mathbb{C}\left[y_{1}, \ldots, y_{j}\right]_{d}, \quad \text { and } \\
W_{x}, W_{y} & \neq 0 .
\end{aligned}
$$

We denote the dual rings $T^{\alpha}=\mathbb{C}\left[\alpha_{1}, \ldots, \alpha_{i}\right], T^{\beta}=\mathbb{C}\left[\beta_{1}, \ldots, \beta_{j}\right], T=T^{\alpha} \otimes$ $T^{\beta}$.

We have $W_{x}^{\perp} \cap W_{y}^{\perp} \subseteq W^{\perp}$. If $0 \leq k \leq d$ and $\Theta \in\left(W^{\perp}\right)_{k}$, then for every $F \in$ $W$, say $F=G-H$ where $G \in W_{x}, H \in W_{y}$, we have $\left.\Theta\right\lrcorner(G-H)=0=$ $\Theta\lrcorner G-\Theta\lrcorner H$. So $\Theta\lrcorner G \in S_{d-k}^{x}$ and $\left.\Theta\right\lrcorner H \in S_{d-k}^{y}$ are equal, which implies $\Theta\lrcorner G=\Theta\lrcorner H=0$ or $d-k=0$. Thus, $\left(W_{x}^{\perp} \cap W_{y}^{\perp}\right)_{k}=W_{k}^{\perp}$ for $0 \leq k<d$. 
Let $F^{1}, \ldots, F^{s}$ be a basis for $W$ and for each $i$, and let $F^{i}=F_{x}^{i}-F_{y}^{i}, F_{x}^{i} \in W_{x}$, $F_{y}^{i} \in W_{y}$. For $1 \leq j \leq s$, let $\delta_{x, j} \in T_{d}^{x}$ be such that $\left.\delta_{x, j}\right\lrcorner F_{x}^{i}=1$ if $i=j$ and 0 if $i \neq j$. Similarly, let $\delta_{y, j} \in T_{d}^{y}$ be such that $\left.\delta_{y, j}\right\lrcorner F_{y}^{i}=1$ if $i=j$ and 0 if $i \neq j$. There are such elements by the linear independence of the $F_{x}^{i}$ and $F_{y}^{i}$. Let $\Delta_{j}=$ $\delta_{x, j}+\delta_{y, j}$. Then $\left.\Delta_{j}\right\lrcorner F^{i}=0$ for each $i$, so $\Delta_{j} \in W^{\perp}$, but $\left.\left.\Delta_{j}\right\lrcorner F_{x}^{j}=\Delta_{j}\right\lrcorner F_{y}^{j}=1$, so $\Delta_{j} \notin W_{x}^{\perp} \cap W_{y}^{\perp}$. Hence, each $\Delta_{j}$ is a minimal generator of $W^{\perp}$. The $\Delta_{j}$ are linearly independent since if $\sum a_{j} \Delta_{j}=0$, then $\left.a_{i}=\left(\sum a_{j} \Delta_{j}\right)\right\lrcorner F_{x}^{i}=0$ for each $i$.

Next we give a generalization of Proposition 1.6.

Proposition 6.4. Let $W \subseteq S^{d} V$. Then $W^{\perp}$ has a minimal generator of degree $d+1$ if and only if there is a nonzero $(d+1)$-form $G \in S^{d+1} V$ such that $\left.T_{1}\right\lrcorner G \subseteq W$.

Proof. First, suppose that $W^{\perp}$ has a minimal generator of degree $d+1$. Let $I \subset$ $W^{\perp}$ be the ideal generated by elements of degree $\leq d$. Then $I_{d+1} \neq T_{d+1}$. Let $G \in S^{d+1} V$ be a nonzero element annihilated by $I_{d+1}$. Since $G$ is annihilated by $I_{d+1}, G$ is annihilated by $I$, so $\left(W^{\perp}\right)_{d}=I_{d} \subseteq\left(G^{\perp}\right)_{d}$. If $\left.F \in T_{1}\right\lrcorner G$, then $G^{\perp} \subseteq F^{\perp}$. In particular, $\left(W^{\perp}\right)_{d} \subseteq F^{\perp}$, so $F \in W$. This shows that $\left.T_{1}\right\lrcorner G \subseteq W$.

Conversely, suppose that $G \in S^{d+1} V$ is a nonzero $(d+1)$-form such that $\left.T_{1}\right\lrcorner$ $G \subseteq W$. As before, let $I \subseteq W^{\perp}$ be the ideal generated by elements of degree $\leq d$. In particular, $\left.I_{d} \subseteq\left(W^{\perp}\right)_{d} \subseteq\left(\left(T_{1}\right\lrcorner G\right)^{\perp}\right)_{d}$. Then $I_{d+1} \subseteq\left(G^{\perp}\right)_{d+1}$ : indeed, if $\Theta=$ $\sum \alpha_{i} \theta_{i}$, each $\theta_{i} \in I_{d}$, then $\left.\left.\left.\Theta\right\lrcorner G=\sum \theta_{i}\right\lrcorner\left(\alpha_{i}\right\lrcorner G\right)=0$ since each $\left.\alpha_{i}\right\lrcorner G \in W$ and each $\theta_{i} \in I_{d} \subseteq\left(W^{\perp}\right)_{d}$. In particular, $I_{d+1} \neq T_{d+1}=\left(W^{\perp}\right)_{d+1}$. So $W^{\perp}$ has a minimal generator of degree $d+1$.

Recall that the $r$ th prolongation of $W \subseteq S^{d} V$ is the set of $(d+r)$-forms $G$ such that $\left.T^{r-d}\right\lrcorner G \subseteq W$ [49, Def. 1.1]. Proposition 6.4 shows that $W^{\perp}$ has a minimal generator of degree $d+1$ if and only if the first prolongation of $W$ is nonzero.

We give a generalization of Theorem 1.9. First, we generalize Lemma 2.1.

Lemma 6.5. Suppose that $W \subseteq S^{d} V$ and $U \subseteq S^{e} V$ are linear series such that $U^{\perp} \subseteq W^{\perp}$. Then $e \geq d$ and $\left.W \subseteq T_{e-d}\right\lrcorner U$. That is, for every $F \in W$, there are $G \in U$ and $\Theta \in T_{e-d}$ such that $\left.F=\Theta\right\lrcorner G$.

Proof. This follows by the inclusion-reversing part of [18, Thm. 21.6].

Now here is a generalization of Theorem 1.9.

Proposition 6.6. Let $W \subseteq S^{d} V$ be a linear series, and $n=\operatorname{dim} V$. Suppose that $W^{\perp}$ has minimal generators $\Theta_{1}, \ldots, \Theta_{s}$ such that $\operatorname{deg} \Theta_{i}=d_{i}$ for each $i$, and $d_{1} \leq \cdots \leq d_{s}$. Let $\delta$ be an integer such that the ideal $\left(W^{\perp}\right)_{\leq \delta}$ is $\mathfrak{m}$ primary. Assume that $d_{k}=\delta<d_{k+1}$ or $k=s$ and $\delta=d_{s}$; necessarily, $k \geq n$. Then $d \leq d_{k}+d_{k-1}+\cdots+d_{k-n+1}-n$. 
The proof is similar to the proof of Proposition 4.11. As before, we immediately deduce the following:

Corollary 6.7. Let $W \subseteq S^{d} V$ be a linear series. Let $n=\operatorname{dim} V$. Suppose that $W^{\perp}$ is generated in degrees less than or equal to $\delta$. Then $d \leq(\delta-1) n$.

It would be interesting to see if our other results, such as Theorem 1.7, can be generalized to linear series. The proofs we have given have used Gorenstein duality, which is not available since the ideals $W^{\perp}$ are not Gorenstein.

\subsection{Overlapping Sums}

Let $F=G_{1}-G_{2}, G_{i} \in S^{d} V_{i}, G_{i} \neq 0$ for $i=1,2$. Theorem 1.1 shows that if $V_{1} \cap$ $V_{2}=\{0\}$, then $F^{\perp}$ has a minimal generator of degree $d$. Here we are interested in allowing $V_{1} \cap V_{2}$ to be nonzero, so that they form an "almost direct sum" or "overlapping sum". We give a statement for the case $\operatorname{dim}\left(V_{1} \cap V_{2}\right)=1$.

Proposition 6.8. Let $F=G_{1}-G_{2}, G_{i} \in S^{d} V_{i}$, with $G_{i}$ concise in $V_{i}$ for $i=1,2$, and suppose that $V_{1} \cap V_{2}$ is one-dimensional, spanned by $x$. Moreover, suppose that $V_{i} \neq\langle x\rangle$ and $\max \left(\operatorname{deg}_{x}\left(G_{1}\right), \operatorname{deg}_{x}\left(G_{2}\right)\right)<d / 2$. Let $s=\max \{t \mid$ $\left.\left.\left.x^{t} \in T\right\lrcorner G_{1} \cap T\right\lrcorner G_{2}\right\}$. Then $F^{\perp}$ has a minimal generator of degree $d-s$.

Proof. As in the proof of Theorem 1.1, $G_{1}^{\perp} \cap G_{2}^{\perp} \subset F^{\perp}$. We claim that $F_{a}^{\perp}=$ $\left(G_{1}^{\perp} \cap G_{2}^{\perp}\right)_{a}$ for $0 \leq a<d-s$. If $\Theta \in F_{a}^{\perp}$, then $\left.\left.\Theta\right\lrcorner G_{1}=\Theta\right\lrcorner G_{2} \in S^{d-a}\langle x\rangle$, that is, $\left.\Theta\lrcorner G_{1}=\Theta\right\lrcorner G_{2}=c x^{d-a}$ for some scalar $c$. We have $\left.\left.x^{s} \in T\right\lrcorner G_{1} \cap T\right\lrcorner G_{2}$, $\left.\left.x^{s+1} \notin T\right\lrcorner G_{1} \cap T\right\lrcorner G_{2}$; more generally, $\left.\left.x^{k} \in T\right\lrcorner G_{1} \cap T\right\lrcorner G_{2}$ if and only if $k \leq s$. So we must have $c=0$ or $d-a \leq s$, equivalently, $\Theta \in G_{1}^{\perp} \cap G_{2}^{\perp}$ or $a \geq d-s$. This proves the claim.

Note that if $\left.x^{t} \in T\right\lrcorner G_{1}$, then $t \leq \operatorname{deg}_{x}\left(G_{1}\right)$. Thus, $s \leq \operatorname{deg}_{x}\left(G_{1}\right)<d / 2$.

Now there exist $\delta_{1}, \delta_{2} \in T_{d-s}$ such that $\left.\left.\left.\delta_{i}\right\lrcorner G_{i}=x^{s}, \delta_{1}\right\lrcorner G_{2}=\delta_{2}\right\lrcorner G_{1}=0$. To see this, let $V_{1}$ have a basis $\left\{x, y_{1}, \ldots, y_{j}\right\}$, and let $V_{2}$ have a basis $\left\{x, z_{1}, \ldots, z_{k}\right\}$. Let $\left\{\alpha, \beta_{1}, \ldots, \beta_{j}\right\}$ be the dual basis for $V_{1}^{*}$, and $\left\{\alpha, \gamma_{1}, \ldots, \gamma_{k}\right\}$ be the dual basis for $V_{2}^{*}$. Let $T^{\beta}=\mathbb{C}\left[\alpha, \beta_{1}, \ldots, \beta_{j}\right]$ and $T^{\gamma}=\mathbb{C}\left[\alpha, \gamma_{1}, \ldots, \gamma_{k}\right]$. We have $T=\mathbb{C}\left[\alpha, \beta_{1}, \ldots, \beta_{j}, \gamma_{1}, \ldots, \gamma_{k}\right]$. There is a $\delta_{1}^{\prime} \in T_{d-s}$ such that $\left.\delta_{1}^{\prime}\right\lrcorner G_{1}=x^{s}$. Since every term of $\delta_{1}^{\prime}$ that involves a $\gamma_{i}$ annihilates $G_{1}$, we can delete those terms to get an element $\delta_{1} \in T_{d-s}^{\beta}$ such that $\left.\delta_{1}\right\lrcorner G_{1}=x^{s}$. Every term of $\delta_{1}$ that has a $\beta_{i}$ annihilates $G_{2}$. If $\delta_{1}$ has a term $c \alpha^{d-s}, c \neq 0$, then by the hypothesis $\operatorname{deg}_{x}\left(G_{2}\right)<d / 2<d-s$ we see that this term also annihilates $G_{2}$. So $\delta_{1} G_{2}=0$, as desired. It is similar to produce $\delta_{2}$.

Let $\Delta=\delta_{1}+\delta_{2}$. Then $\Delta \in F_{d-s}^{\perp}$, but $\Delta \notin G_{1}^{\perp} \cap G_{2}^{\perp}$. Hence, $\Delta$ is a minimal generator of $F^{\perp}$ : it cannot be generated in lower degrees since all elements in lower degrees lie in $G_{1}^{\perp} \cap G_{2}^{\perp}$.

Corollary 6.9. Let $F=G_{1}-G_{2}, G_{i} \in S^{d} V_{i}, G_{i}$ concise in $V_{i}$ for $i=1,2$, and suppose that $V_{1} \cap V_{2}$ is one-dimensional, spanned by $x$. Moreover, suppose $V_{i} \neq$ 
$\langle x\rangle$ and $t=\max \left(\operatorname{deg}_{x}\left(G_{1}\right), \operatorname{deg}_{x}\left(G_{2}\right)\right)<d / 2$. Then $F^{\perp}$ has a minimal generator of degree at least $d-t$, in particular, strictly greater than $d / 2$.

It would also be interesting to investigate cases with larger overlap or with more than two overlapping summands.

\subsection{Other Base Fields}

Most of our results, including results overlapping with [33], also hold over any algebraically closed field $\mathbb{k}$. The main difference is that we need to consider $S=\mathbb{k}\left[x_{1}, \ldots, x_{n}\right]^{D P}$ to be the divided power algebra, rather than the polynomial ring, and the apolarity action of $T$ on $S$ is now as if the differentiation was very naive: $\left.\alpha_{i}\right\lrcorner x_{i}{ }^{(d)}=x_{i}{ }^{(d-1)}$ (no $d$ coefficient); see [29, App. A], [18, Sect. A2.4]. All occurrences of powers of linear forms in $S$ should now be replaced by the divided powers, for instance, $x^{d-1} y$ should be $x^{(d-1)} y$, and so on. In particular, the Veronese embedding $v_{d}: \mathbb{P} V \rightarrow \mathbb{P}\left(S^{d} V\right)$ is now $v_{d}([x])=\left[x^{(d)}\right]$, and the Waring rank is computed with respect to the image of $v_{d}$ (and the border rank, cactus rank, etc.).

In this setup, Theorems 1.1, 1.7, and 1.9 and their proofs remain valid over any algebraically closed base field $\mathbb{k}$ of any characteristic; in particular, direct sums and their concise limits have equipotent apolar generators; any concise dividedpowers form with an equipotent apolar generator is a limit of direct sums; and the bound $d \leq(\delta-1) n$ relating the greatest degree $\delta$ of apolar generators and the degree $d$ of the form is valid. Proposition 1.6 only requires the change of $\ell^{d}$ into $\ell^{(d)}$, so that forms of degree $d$ with apolar generator in degree $d+1$ essentially depend on just one variable. Proposition 2.12 is proved over any field in [51].

Theorem 1.8 consists of two parts. The first consists of Theorem 5.19 (in three variables, all limits of direct sums are apolar limits of direct sums) and Proposition 5.2 (all limits of cubics are apolar limits), which are valid with no significant change to the statements or proofs. In fact the proof of Theorem 5.19 becomes slightly simpler as all coefficients like $\frac{d !}{k !}$ or $\left(\begin{array}{c}k \\ q-i\end{array}\right)$ are replaced with just 1 and in the end the matrices $M$ and $N$ are just equal. The second part is Proposition 5.17 (there exists a limit of direct sums, which is not an apolar limit of direct sums). To prove this statement, we used results from [8], which is written over $\mathbb{C}$, so Proposition 5.17 is not proven over $\mathbb{k}$. Similarly, the other results presented in Sections 5.1 and 5.2 also depend on [8]. However, the first and second authors believe that the results of [8] used in this article can be generalized to any characteristic.

Section 2.5 and Table 1 describe the behavior and classification of plane cubics. In positive characteristics, these are different, particularly the cases of characteristics 2 and 3. The numerous examples throughout the paper might be valid only in some characteristics, whereas in the other characteristics, they need to be appropriately adjusted. The exact value of the integer $n_{0, \mathbb{k}}$ (the least length of a nonsmoothable Gorenstein scheme) might be different in positive characteristics, particularly in characteristics 2 and 3. Similarly, the set of integers $n_{1}$ (the lengths 
of uncleavable Gorenstein schemes) may be different in positive characteristics, particularly in characteristics 2 and 3 . It is beyond the scope of this paper to pursue these issues, but see the references mentioned in Section 5.2. No other changes are needed to make this paper valid over any algebraically closed field.

ACKNOWLEDGMENTs. As indicated by appropriate citations, many of the statements in this article are either a part of the third author's Ph.D. thesis [33], or they are similar to statements in that thesis. We thank Sepideh Shafiei for suggesting to us the problem of direct sum decomposability of determinants and for sharing with us her papers $[47 ; 48]$ while they were in preparation. We thank Joachim Jelisiejew, Grzegorz Kapustka, Jan O. Kleppe, Robert Lazarsfeld, and Anna Otwinowska for helpful comments and Kristian Ranestad for connecting the unpublished thesis of the third author with the work of the other authors. We especially thank the anonymous referee for unusually thorough reviews, which included a number of helpful suggestions. The computer algebra software packages Macaulay2 [24] and Magma [6] were useful in compiling Table 1 and in calculations of examples.

\section{References}

[1] H. Ananthnarayan, L. L. Avramov, and W. Frank Moore, Connected sums of Gorenstein local rings, J. Reine Angew. Math. 667 (2012), 149-176.

[2] H. Ananthnarayan, E. Celikbas, and Z. Yang, Decomposing Gorenstein rings as connected sums, 2014, arXiv:1406.7600.

[3] A. Bernardi, A. Gimigliano, and M. Idà, Computing symmetric rank for symmetric tensors, J. Symbolic Comput. 46 (2011), no. 1, 34-53.

[4] C. Bertone, F. Cioffi, and M. Roggero, A division algorithm in an affine framework for flat families covering Hilbert schemes, 2012, arXiv:1211.7264.

[5] M. Boratyński and S. Greco, Hilbert functions and Betti numbers in a flat family, Ann. Mat. Pura Appl. (4) 142 (1985), 277-292.

[6] W. Bosma, J. Cannon, and C. Playoust, The Magma algebra system. I. The user language, J. Symbolic Comput. 24 (1997), no. 3-4, 235-265.

[7] D. A. Buchsbaum and D. Eisenbud, Algebra structures for finite free resolutions, and some structure theorems for ideals of codimension 3, Amer. J. Math. 99 (1977), no. 3, 447-485.

[8] W. Buczyńska and J. Buczyński, Secant varieties to high degree Veronese reembeddings, catalecticant matrices and smoothable Gorenstein schemes, J. Algebraic Geom. 23 (2014), no. 1, 63-90.

[9] J. Buczyński, A. Ginensky, and J. M. Landsberg, Determinantal equations for secant varieties and the Eisenbud-Koh-Stillman conjecture, J. Lond. Math. Soc. (2) 88 (2013), no. 1, 1-24.

[10] E. Carlini, Reducing the number of variables of a polynomial, Algebraic geometry and geometric modeling, Math. Vis., pp. 237-247, Springer, Berlin, 2006.

[11] G. Casnati, J. Jelisiejew, and R. Notari, Irreducibility of the Gorenstein loci of Hilbert schemes via ray families, Algebra Number Theory (to appear).

[12] _ On the rationality of Poincaré series of Gorenstein algebras via Macaulay's correspondence, Rocky Mountain J. Math. (to appear). 
[13] G. Casnati and R. Notari, On the irreducibility and the singularities of the Gorenstein locus of the punctual Hilbert scheme of degree 10, J. Pure Appl. Algebra 215 (2011), no. 6, 1243-1254.

[14] On the Gorenstein locus of the punctual Hilbert scheme of degree 11, J. Pure Appl. Algebra 218 (2014), no. 9, 1635-1651.

[15] J. Chipalkatti, Apolar schemes of algebraic forms, Canad. J. Math. 58 (2006), no. 3, 476-491.

[16] A. Dimca and A. Némethi, Thom-Sebastiani construction and monodromy of polynomials, Tr. Mat. Inst. Steklova 238 (2002), 106-123.

[17] R. Ehrenborg and G.-C. Rota, Apolarity and canonical forms for homogeneous polynomials, European J. Combin. 14 (1993), no. 3, 157-181.

[18] D. Eisenbud, Commutative algebra with a view toward algebraic geometry, Grad. Texts in Math., 150, Springer-Verlag, New York, 1995.

[19] _ The geometry of syzygies: a second course in commutative algebra and algebraic geometry, Grad. Texts in Math., 229, Springer-Verlag, New York, 2005.

[20] S. El Khoury, A. V. Jayanthan, and H. Srinivasan, On the number of generators of ideals defining Gorenstein Artin algebras with Hilbert function $(1, n+1$, $\left.1+\left(\begin{array}{c}n+1 \\ 2\end{array}\right), \ldots,\left(\begin{array}{c}n+1 \\ 2\end{array}\right)+1, n+1,1\right), 2013$, arXiv:1304.0305.

[21] S. El Khoury and H. Srinivasan, A class of Gorenstein Artin algebras of embedding dimension four, Comm. Algebra 37 (2009), no. 9, 3259-3277.

[22] J.-P. Françoise, Systèmes maximaux d'une singularité quasi homogène, C. R. Acad. Sci. Paris Sér. A-B 290 (1980), no. 22, A1061-A1064.

[23] A. V. Geramita, Inverse systems of fat points: Waring's problem, secant varieties of Veronese varieties and parameter spaces for Gorenstein ideals, The Curves Seminar at Queen's, Vol. X, Kingston, ON, 1995, Queen's Papers in Pure and Appl. Math., 102, pp. 2-114, Queen's Univ., Kingston, ON, 1996.

[24] D. R. Grayson and M. E. Stillman, Macaulay2, a software system for research in algebraic geometry, 〈http://www.math.uiuc.edu/Macaulay2/〉.

[25] M. L. Green, Generic initial ideals, Six lectures on commutative algebra, Progr. Math., 166, pp. 119-186, Birkhäuser, Basel, 1998.

[26] R. Hartshorne, Algebraic geometry, Grad. Texts in Math., 52, Springer-Verlag, New York, 1977.

[27] A. Iarrobino, Deforming complete intersection Artin algebras. Appendix: Hilbert function of $\mathbf{C}[x, y] / I$, Singularities, Part 1, Arcata, Calif., 1981, Proc. Sympos. Pure Math., 40, pp. 593-608, Amer. Math. Soc., Providence, RI, 1983.

[28] Compressed algebras and components of the punctual Hilbert scheme, A1gebraic geometry, Sitges (Barcelona), 1983, Lecture Notes in Math., 1124, pp. 146165, Springer, Berlin, 1985.

[29] A. Iarrobino and V. Kanev, Power sums, Gorenstein algebras, and determinantal loci, Lecture Notes in Math., 1721, Springer-Verlag, Berlin, 1999. Appendix C by A. Iarrobino and Steven L. Kleiman.

[30] A. Iarrobino and H. Srinivasan, Artinian Gorenstein algebras of embedding dimension four: components of $\mathbb{P G o r}(H)$ for $H=(1,4,7, \ldots, 1)$, J. Pure Appl. Algebra 201 (2005), no. 1-3, 62-96.

[31] J. Jelisiejew, Local finite-dimensional Gorenstein $k$-algebras having Hilbert function $(1,5,5,1)$ are smoothable, J. Algebra Appl. 13 (2014), no. 8, 1450056.

[32] J.-P. Jouanolou, Théorèmes de Bertini et applications, Progr. Math., 42, Birkhäuser Boston Inc., Boston, MA, 1983. 
[33] J. Kleppe, Additive splittings of homogeneous polynomials, Ph.D. thesis, Univ. Oslo, 2005, arXiv:1307.3532.

[34] J. O. Kleppe, The Hilbert scheme of Buchsbaum space curves, Ann. Inst. Fourier (Grenoble) 62 (2012), no. 6, 2099-2130.

[35] J. M. Landsberg and Z. Teitler, On the ranks and border ranks of symmetric tensors, Found. Comput. Math. 10 (2010), no. 3, 339-366.

[36] F. S. Macaulay, The algebraic theory of modular systems, Cambridge Math. Lib., Cambridge University Press, Cambridge, 1994. Revised reprint of the 1916 original, with an introduction by Paul Roberts.

[37] D. M. Meyer and L. Smith, Poincaré duality algebras, Macaulay's dual systems, and Steenrod operations, Cambridge Tracts in Math., 167, Cambridge University Press, Cambridge, 2005.

[38] J. C. Migliore, The geometry of Hilbert functions, Syzygies and Hilbert functions, Lect. Notes Pure Appl. Math., 254, pp. 179-208, Chapman \& Hall/CRC, Boca Raton, FL, 2007.

[39] A. Némethi, Generalized local and global Sebastiani-Thom type theorems, Compos. Math. 80 (1991), no. 1, 1-14.

[40] _ Global Sebastiani-Thom theorem for polynomial maps, J. Math. Soc. Japan 43 (1991), no. 2, 213-218.

[41] I. Peeva, Consecutive cancellations in Betti numbers, Proc. Amer. Math. Soc. 132 (2004), no. 12, 3503-3507.

[42] A. Ragusa and G. Zappalá, On the reducibility of the postulation Hilbert scheme, Rend. Circ. Mat. Palermo (2) 53 (2004), no. 3, 401-406.

[43] K. Ranestad and F.-O. Schreyer, On the rank of a symmetric form, J. Algebra 346 (2011), 340-342.

[44] B. Reznick, An inequality for products of polynomials, Proc. Amer. Math. Soc. 117 (1993), no. 4, 1063-1073.

[45] M. Sebastiani and R. Thom, Un résultat sur la monodromie, Invent. Math. 13 (1971), 90-96.

[46] I. R. Shafarevich, Deformations of commutative algebras of class 2, Algebra i Analiz 2 (1990), no. 6, 178-196.

[47] S. M. Shafiei, Apolarity for determinants and permanents of generic matrices, J. Commut. Algebra 7 (2015), no. 1, 89-123.

[48] __ Apolarity for determinants and permanents of generic symmetric matrices, 2013, arXiv:1303.1860.

[49] J. Sidman and S. Sullivant, Prolongations and computational algebra, Canad. J. Math. 61 (2009), no. 4, 930-949.

[50] L. Smith and R. E. Stong, Poincaré duality algebras mod two, Adv. Math. 225 (2010), no. 4, 1929-1985.

[51] _ Projective bundle ideals and Poincaré duality algebras, J. Pure Appl. Algebra 215 (2011), no. 4, 609-627.

[52] R. P. Stanley, Hilbert functions of graded algebras, Advances in Math. 28 (1978), no. $1,57-83$.

[53] J. J. Sylvester, An essay on canonical forms, supplement to a sketch of a memoir on elimination, transformation and canonical forms, The collected mathematical papers of James Joseph Sylvester, Vol. 1, pp. 203-216, Chelsea, New York, 1973. Originally published by Cambridge University Press, Cambridge, 1904.

[54] R. Thom, Problèmes rencontrés dans mon parcours mathématique: un bilan, Publ. Math. Inst. Hautes Études Sci. 70 (1989), 199-214. 
[55] K. Ueda and M. Yoshinaga, Logarithmic vector fields along smooth divisors in projective spaces, Hokkaido Math. J. 38 (2009), no. 3, 409-415.

W. Buczyńska

Institute of Mathematics of the

Polish Academy of Sciences

ul. Śniadeckich 8

P.O. Box 21

00-956 Warszawa

Poland

wkrych@mimuw.edu.pl

J. Kleppe

Faculty of Technology and Maritime Sciences

Buskerud and Vestfold University College

P.O. Box 235

3603 Kongsberg

Norway
J. Buczyński

Institute of Mathematics of the

Polish Academy of Sciences

ul. Śniadeckich 8

P.O. Box 21

00-956 Warszawa

Poland

and

Faculty of Mathematics, Computer

Science and Mechanics

University of Warsaw

ul. Banacha 2

02-097 Warszawa

Poland

jabu@mimuw.edu.pl

Z. Teitler

Department of Mathematics

Boise State University

1910 University Drive

Boise, ID 83725-1555

USA

zteitler@boisestate.edu

johannes.kleppe@hbv.no 